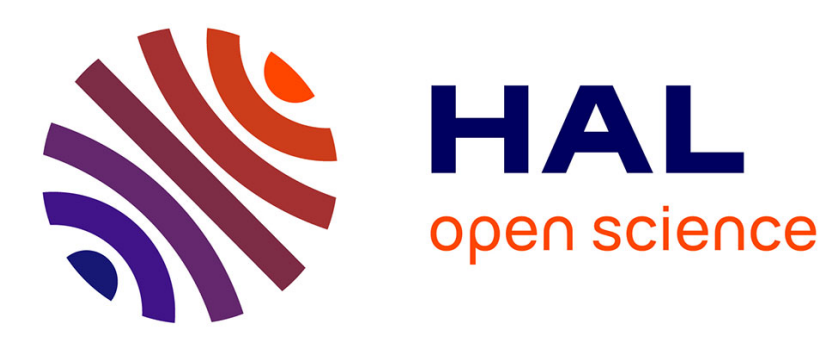

\title{
Sur le rapport du poids de l'encéphale avec la grandeur du corps chez les mammifères
}

\author{
Eugène Dubois de La Haye
}

\section{To cite this version:}

Eugène Dubois de La Haye. Sur le rapport du poids de l'encéphale avec la grandeur du corps chez les mammifères. Bulletins et Mémoires de la Société d'anthropologie de Paris, 1897, pp.337-376. hal-03581741

\section{HAL Id: hal-03581741 \\ https://hal.science/hal-03581741}

Submitted on 20 Feb 2022

HAL is a multi-disciplinary open access archive for the deposit and dissemination of scientific research documents, whether they are published or not. The documents may come from teaching and research institutions in France or abroad, or from public or private research centers.
L'archive ouverte pluridisciplinaire HAL, est destinée au dépôt et à la diffusion de documents scientifiques de niveau recherche, publiés ou non, émanant des établissements d'enseignement et de recherche français ou étrangers, des laboratoires publics ou privés.

\section{(c)(2)}

Distributed under a Creative Commons CCO - Public Domain Dedication| 4.0 International 
Sur le rapport du poids de l'encéphale avec la grandeur du corps

\author{
ol. Jo Par Eugène Dubors (de La Haye). \\ (Mémoire présenté par M. Manouvrier).
}

Le système nerveux central des animaux a gagné en complexité et en volume à mesure que leurs relations avec le monde extérieur se sont multipliées. Chez les vertébrés, des poissons aux mammifères, la partie supérieurement organisée, l'encéphale, devient plus pesant en comparaison de la parlie moins compliquée, la moelle épinière. Ce sont les parties les plus différenciées de l'encéphale, les grands hémisphères cérébraux, qui, surlout vont surpasser les autres. Les mammifères ont l'encéphale beaucoup plus volumineux et plus perfectionné que tous les autres animaux et montrent en quelque mesure suivant le degré de l'ordre auquel ils appartiennent un développement ascendant. Ils ont acquis cette organisation supérieure progressivement. Marsh et autres, ont démontré que les dimensions du cerveau chez les mammifères de l'époque éocène sont généralement moindres que chez les formes apparentées du Néo-tertiaire ou de l'époque actuelle ${ }^{2}$. Les mammifères à placenta de tous les ordres dans le plus ancien terrain éocène ont la cavité cranienne extrêmement petite. Dans des animaux appartenant a l'un de ces ordres l'encéphale était plus mince que la moelle épinière, à ce point mème, qu'on aurait pu facilement l'extraire du crane par le trou occipital et par le canal vertébral. Marsh démontre en mème temps que ce sont surtout les parties supérieurement organisées de l'encéphale, les hémisphères cérébraux, qui, chez ces plus anciens mammifères placentaires, étaient très peu développées, d'où il résulte que ces formes sont même moins éloignées sous ce point des reptiles que des mammifères placentaires récents.

Ce développement progressif de l'encéphale, en organisation et en volume, chez les vertébrés et principalement chez les mammifères, ainsi que le volume et la complexité en structure remarquables qu'il atteint finalement chez l'homme, démontrent que

1 Publié déjà en substance en hollandais dans les Mémoires de l'Académie royate des Sciences d'Amsterdam, t. V, n 10, avril 1897.

2 A. Gaudsy, Essai de paléontologie philosophique- Daris, 1896, p. 147-154. PALEONVIGE BGIE)
HUMAINE
PARIS

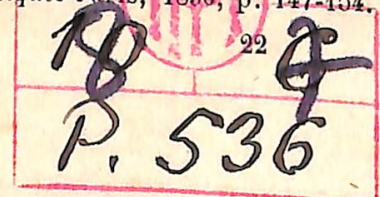


dans cet organe aussi le développement de la fonction détermine la complication de la structure et la quantité. Aurun animal de même grandeur ne possède une quantité d'encéphale comparable à celle de l'homme. Les grands singes anthropoïdes, dont le poids du corps équivaut à celui de l'homme, n'atteignent pas mème $1 / 3$, et les chiens du mème poids $1 / 10$, quant à l'encéphale, du poids de celui de l'homme ${ }^{1}$. Le cheval, qui pèse en moyenne six fois autant que l'homme, a un encéphale qui reste au-dessous de la moitié du poids de celui de l'homme. Seuls, l'éléphant, les plus grandes baleines et la Rhytine (Rhytina stelleri), animal disparu au siècle dernier,l'emportent sur l'homme, quant au poids absolu de l'encéphale. Chez l'éléphant, en effet, l'encé5 -fois, et chez los 4 fois, chez les plus grandes baleines certes 1 et $1 / 2$ fois le poids De deux a poids moyen de celui de l'homme.

à un degré très différ la taille est la même mais qui se trouvent l'échelle possède toujours d'organisation, le plus haut placé de le Siamang (Hylobates syndacéphale le plus pesant. Ainsi, chez de $1 / 73$, chez le sates syndactylus), le poids de l'encéphale est Civette (Vivërra civetta) $1 / 20$ nnopithecus maurus) $1 / 126$, chez la javanica) $1 / 615$, chez tous $1 / 202$, chez le Pangolin javanais (Manis à peu près le mème. L'espèce d'Oux le poids du corps étant vulgairement sous le nom desè d'Ouistiti (Midas rosalia), connu sur 26 grammes de poids de singe-lion a 1 gramme d'encéphale poids est à peu près le même, corps, tandis que l'écureuil, dont le d'encéphale sur 6 grammes de possède pas plus que 1 gramme

De deux animaux

sont du même type, mais rapport à l'organisation cérébrale, plus grand animal a aussi t'enilles très différentes, toujours le par exemple, a un cerveau 7 fois phale le plus pesant. Le lion, naire; celui du surmulot (le fois plus pesant que le chat ordicelui de la souris; celui du Beisa, antile pèse $51 / 2$ fois autant que à celle du cerf commun, atleint, antilope dont la taille est égale lope pygmée (Cephalophus maxwelli) corps qu'un lièvre.

de deux facteurs:

${ }^{1}$ Voir les tables ci-dessous. Toujours des anin leur crue normale sont comparés.
10 Du degré de développement que l'encéphale a atteint comme organe, en d'autres termes de la céphalisation du système nerveux central;

$2^{\circ}$ De la grandeur du corps.

On ne peut cependant pas mettre simplement pour la grandeur du corps, son poids. Car si l'on compare le poids de l'encéphale avec celui du corps chez des animaux identiquement organisés on constate que le poids du cerveau ne constitue nullement toujours la même fraclion du poids du corps. Universellement chez les petits animaux le poids de l'encéphale est relativement plus élevé. Le poids relatif de l'encéphale est chez le chat de $1 / 106$, le lion $1 / 546$, chez la souris $1 / 49$, le surmulot $1 / 190$, chez l'écureuil commun 1/65, le grand écureuil de Java (Sciurus bicolor) 1/116, chez l'antilope pyomée $1 / 88$, le Beisa $1 / 382$. Le poids relatif de l'homme qui est de $1 / 46$ à 1/45̆, est dépassé par celui de quelques animaux dont le poids du corps est minime : par la musaraigne, dont le poids relatif de l'encéphale est de $1 / 23$, le singe-lion qui a un poids encéphalique $1 / 26$, le Tupaja javanais qui a $1 / 41$ d'encéphale, la chauve-souris à moustaches (Vespertılio mystacinus) qui en a $1 / 42$; cependant parmi ceux-ci, chez la musaraigne et la chauve-souris l'encéphale a une organisation fort inférieure.

Quoiqu'il soit connu depuis longtemps que la grandeur du corps influence le poids de l'encéphale et que Cuvier pouvait déjà dire que. toutes choses égales, les pelits animaux possèdent proportionnellement les plus grands encéphales, la raison de cette influence est restée encore dans l'obscurité. La cause en réside sursurtout en ce que dans la plupart des anciennes déterminations de poids, il n'est pas sulfisamment tenu compte du fait que l'encéphale augmente moins en poids avec l'age que le reste du corps. Ghez l'homme il atteint déjà vers la neuvième année à peu près le mème poids que chez l'adulte, tandis que le poids du corps augmente encore plus du double. La même chose se constate, comme l'a démontré $M$. Weber, chez tous les mammifères; l'encéphale a achevé de croître beaucoup plus tôt que les autres parties du corps. C'est pourquoi il faut comparer les animaux adultes. Les animaux à comparer doivent aussi ètre autant que possible dans le même état de nutrition, et on ne doit pas seulement tenir compte du poids relatif, mais aussi du poids absolu de l'encéphale et de celıi du corps. Les déterminations du poids doivent ètre faites sur des encéphales à l'état frais, et enfin-mais ceci restera 
encore longtemps un pium desideratum - on devrait posséder pour chaque espèce d'animaux adultes des déterminations moyennes de poids d'un grand nombre d'individus. Les anciennes déterminations de Cuvier et de Leuret ne peuvent par là presque pas servir, et de mème quelques autres séries de déterminations n'ont pu apporter que peu de lumière dans l'obscurité qui entoure ce sujet. Quelques-unes, entre autres celles de Colin, par là même qu'elles ont rapport surtout aux animaux domestiques, qui pour la plupart présentent à l'examen de grandes différences dans le poids du corps avec leurs congénères vivant en liberté.

C'est le grand mérite de M. Max Weber ${ }^{1}$, d'avoir donné des poids exacts du cerveau et du corps chez un grand nombre d'espèces de de telles données, élerminées, et d'avoir fourni en mème temps adulte ou jeune et au'on peut dans presque tous les cas juger l'état tion de l'animal en condition de nutrifinitivement démontré qu'il existe ré entre le poids de l'encéphale et celui disproportion petits mammifères possèdent cen sens que les plus d'encéphale que les mammifert au poids de leur corps groupe systématique, mais qui ont ères appartenanl au mème point de départ fixe est en mème corps plus grand. Par là un manière précise dont le poids du temps donné pour juger de la du corps. Weber ayant définitivement dépend de la grandeur temps cause d'un assez grand embar prouvé le fait, qui fut longque pour le cerveau aussi il doit pour ceux qui admettaient masse et la fonction de l'organe, $\mathrm{y}$ avoir un rapport entre la les données nécessaires pour mettre ficuré en même temps prochant ces données des faits élabli à cet embarras. En rapstructure du système nerveux élablis aujourd'hui concernant la "facteur somatique ) en question.

Il conduirait trop loi

nières dont on a tâché de le fair exposer les différentes masuccinte des différentes directions jusqu'ici; qu'une exposition suffise. $\quad$

Gratiolet ${ }^{2}$ n'attribuait aucune signification à la quantité céré-

1 Max Weber, Vorstudien über das Hirngewicht a la quantité céré-

abdruck aus Festschrift für Carl Gegenbaur. Leit der Süugethiere. Separat-

2 Leuret et Gratiolet, Anatomie compaur. Leipzig, 1896.

p. 104 sqq. brale, vu que le poids relatif du cerveau de l'homme est dépassé par celui d'un certain nombre de petits animaux et qu'on n'avait pu démontrer que dans l'espèce humaine un plus grand volume cérébral marche toujours de pair avec une plus grande intelligence. Selon lui, il faudrait simplement tenir compte de la forme du cerveau et surtout du développement des circonvolutions.

Dareste $^{1}$ au contraire admettait que le poids relatif plus élevé de l'encéphale chez les petits animaux, en comparaison de celui des plus grands, appartenant à un mème groupe, s'expliquerait par l'intelligence plus grande des premiers. En se référant aux dounées récentes cette interprétation n'est plus admissible, car chez le chat l'encéphale comme organe n'est certes pas cinq fois plus compliqué que chez le lion et chez la souris l'encéphale ne fait pas preuve, par sa fonction, d'une structure quatre fois plus compliqué que chez le rat brun.

Beaucoup ont cru que pour la détermination de la valeur de l'organe il fallait considérer l'encéphale non dans son ensemble mais dans certaines parties spéciales.

Selon d'autres la quantité de l'encéphale serait en partie déterminée par la masse des organes du corps, mais une autre partie, règlant les processus psychiques, serait dans ses fonctions plus ou moins indépendante de la quantité. Les animaux de grande taille devraient surtout le grand poids de leur corps à des organes dont la masse est de peu d'importance pour la quantilé de l'encéphale, tandis que, chez les petits animaux, ce sont précisément les organes influant sur la quantité de l'encéphale qui sont relativement très développés. Cette interprétation a trouvé en M. Manouvrier son principal avocat. Les recherches de ce savant sont certainement les plus profondes que nous possédons sur ce sujet, et ses résultats sont le mieux en harmonie avec les idées nouvelles sur la structure de l'encéphale. Son opinion : «Dans des espèces extrèmement petites le développement relatif des organes les plus en rapport avec l'encéphale devient tel, que, mème étant moins nombreux et moins compliqués que dans d'autres espèces de grande taille, ils égalent ou surpassent en poids et en étendue les organes dont l'accroissement influence le moins le cerveau. Il en résulte que ces animaux, en dépit de l'infériorité de leur type acquièrent

${ }^{1}$ Sur les rapports de la masse encéphalique avee le developpement de 'intelligence. Bull. de la Soc. d'Anthrop. de Paris, 1862. 
un poids cérébral relatif dépassant celui d'animaux bien supédification pour la mettre $)^{1}$, ne devra subir qu'une légère modéterminations de $M$ Tes résultats auxquels les tuelles sur la structure duer, rapprochées des connaissances ac-

Il me semble que les conclusione nerveux peuvent conduire. une forme décisive principalement Manouvrier n'ont pu prendre à l'homme, ne pouvant dispent parce qu'il s'est restreint surtout sur les mammifères. Cependant que de très insuffisantes données derniers, par cause des plus en comparant les espèces de ces plus d'espérance à résoudre le différences des nombres il y Brandt cherche l'explication problème q'i nous occupe. grandeur d'un animal et la quans le rapport qui existe entre la qu'il produit. Chez les petits anantité du travail physiologique du corps relativement plus grande é égard à leur surface séquent l'échange de matière a perle de calorique et par conpiration sont plus actives. centres psychiques cérébraux. Laissant hors de consıdération les époque, Brandt admeltait que proprement dits, inconnus à cette sont sous la dépendance que ces procès physiologiques, qui tous avoir par conséquent des cen système nerveux central, doivent procès susnommés sont plus intentant plus développés que les des procès physiologiques plus actifs. Les petits animaux ayant centres nerveux plus développés. Aussi chez donc posséder des petits les procès de la vie animale proprem des animaux plus mène tem la surface exténent dite seraient relamème temps sa surface sensitive et celérieure de l'animal est en grande chez les petits animaux. De ple-ci est relativement plus veuses motrices augmenterait propor le nombre des fibres nerface de la section et non suivant portionnellement suivant la surBischoff ${ }^{3}$ admet ave: Brandt que des muscles. par suite de leur échange de matière chez les petits animaux, tivement plus grand doit présider aur intense, un centre relacerveau.

1 L. Manouvrier, sur l'interprétalion de

$2 \circ$ série, $t$. III (188 particulier. Mémoires de quantité dans l'encéphale et 2 A. Brandt, sur le 137-326.

férents animaux, Bulletin de du poids du cerveau a

t. XL, 2, p. 525-543.

3 Th. L. W. v. Bischoff, Das Hirngewicht des Menschen. Bonn 1880 ,
Snell 1 aussi attribue le poids relatif plus élevé de l'encéphale des petits mammifères à ce que l'encépale outre ses fonctions psychiques doit remplir aussi une fonction pour l'échange de matière, donc pour une fin purement somatique. Et ces processus de l'échange de matière sont relativement plus grands à mesure que le corps de l'animal est plus petit, parce que les petits animaux ont par rapport au poids du corps une plus grande surface que les animaux de grande taille. Selon Snell, le poids de l'encéphale augmente toutefois en plus forte raison que la surface du corps, vu que la masse de l'animal exerce aussi de l'influence sur la somme de travail qui produit l'échange de matière. Snell veut donc interpréter le fait paradoxal à peu près de la mème manière que Brandt l'avait fait. Il voit dans l'intensité de l'échange de matière mème un facteur plus important que celui-ci. Son mérite spécial est toutefois d'avoir formulé l'hypothèse admise par Brandt déjà : que c'est la surface qui dans la grandeur du corps détermine le poids de l'encéphale - d'une manière plus mathématique.

L'interprétation de Fürbringer ${ }^{2}$ est d'accord avec celle de Brandt. Il tend à attribuer le plus grand volume de l'encéphale des petits animaux, du moins en partie, en rapport avec l'échange plus actif de matière, à un centre calorique plus important de l'encéphale. Il croit aussi devoir altribuer à un moindre développement du centre thermique les petites dimensions de l'encéphale des mammifères du plus ancien terrain éocène et des oiseaux mésozoïques.

Les expériences de Regnault et Reiset nous apprenaient déjà et c'est démonlré définitivement par les expériences de Rubner ${ }^{3}$ que l'échange de matière chez des animaux d'ailleurs comparables n'est pas proportionnée au poids de leur corps, mais aux surfaces. Aussi on peut déduire des chiffres donnés par M. Weber, qu'il existe réellement une approximation à un tel rapport entre la surface du corps et le poids du cérveau que présumaient Brandt ét Snell, cependant le poids de l'encéphale ne croit aucunement proportionnellement à la surface du corps. Aussi ce rapport à une toute

1 Archiv für Psychiatrie, 1891 Bd. 23, p. 436-446 et Sitzungsberichte der Gesellsch, für Morphologie und Physiologic, München 1891.

2 Max Fürbringer, Untersuchungen zur Morphologie und Systematik der Vögel, II, Amsterdam, 1888, p. 1639-1640.

3 Zeitschrift für Biologie, 1883, Bd. 19, p. 536. 
autre raison que ces auteurs supposaient. Brandt et ses imitateurs ont admis à tort un rapport direct entre deux phénomènes séparés, qui, il est vrai, s'influencent indireclement de part et d'autre. D'après les données établies sur la structure et la fonction de l'encéphale il faut admettre que cet organe ne préside qu'en trés faibles proporlions à l'échange de matière et non principalement comme le supposaient Brandt, Snell et surtout Fürbringer.

Gratiolet avait altribué une grande signification au développement des circonvolutions pour la détermination de développecerveau comme organe. Richard 0wen avait mème, d'après ce principe, réparti les mammifères vivants en trois sous-classes. Quoique le cerveau des mammifères des ordres supérieurs soit en général plus riche en circonvolutions que celui de quelques rapport. Owen devait déjà en ériondant pas de règle fixe sous ce nombre de grands animaux exigeant ses sous-classes excepter bon animaux des Gyrencéphales. C'est aisencéphales, beaucoup de petits présente plus de circonvolutions que que le cerveau de la chèvre près identique du siamang, un que l'encéphale d'un poids à peu cependant comme une règle presque anthropoïde. On remarque misphères du cerveau des grands générale que les giands hécirconvolutions que chez les petits animaux présentent plus de turel. C'est un fait connu depuis animaux du mème groupe nafailement et qu'il ne faut pas perdre de sur le poids relatif de l'encéphale. et petits animaux d'un mème groupe si les cerveaux des grands fait semblables, c'est-à-dire sont différe naturel ne sont pas tout à peut non plus en comparer directementans leurstructure, on ne petites espèces de tous les ordres des le poids. Chez les très singes, les circonvolutions manquent, mammifères, mème des espèces des groupes aussi inférieurs ot chez les plus grandes supiaux, on les rencontre. Elles alteignent Édentés et les Marloppement chez les Célacés. Le cerveaut leur plus grand dévemal de taille égale à celle de l'écureuil, est singe-lion, petit anigibbons ont beaucoup moins de circont parfaitement lisse. Les singes anthropoïdes. Les hémisphères cérébutions que les grands lus javanica), présentent peu de circonvolu coup plus nombreuses et plus développées ; celles-ci sont beau1 Comp. anatomy, vol. III, p. $39, \mathrm{sqq}$. rence analogue se constate dans la comparaison du hyrax et du rhinocéros. La différence du cerveau dans la famille si naturelle des chals consiste principalement en ce que chez les espèces les plus petites les circonvolutions secondaires font défaut ensore ${ }^{1}$, ce qui se constate à l'évidence en comparant le cerveau du chat commun avec celui du tigre. Chez ce dernier cela fait l'impression circonvolutions ayaient dù se plisser con latéral. Parmi les Rongeurs, la celui-ci au capy-

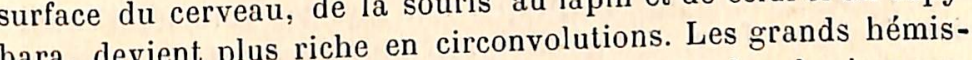
phères du dauphin (Delphinus delphis) ont un nombre de circonvophèr exérés, par exemple de l'Hyperoodon rostratus ${ }^{2}$.

C'est donc un fait acquis que la grandeur du corps influe sur la richesse des circonvolutions. Cependant il ne fautpas exagérer ce influence; nous verrons dans la suite qu'elle trouve une compensation très réelle. Le premier, Baillarger ${ }^{3}$ en a donné cette interprétation que chez les animaux apparentés, mais très différents en grandeur, non seulement le volume, mais certes pas moins la surface du cerveau doit augmenter. Si le cerveau acquiert par exemple un volume 8 fois plus considérable, sa surface devient seulement 4 fois plus grande. Si toutefois la surface doit augmenter dans la mème proportion que le volume, elle doit se plisser; car c'est le seul moyen de s'agrandir. Jelgersma ${ }^{4}$ est évidement indépendamment de Baillarger, arrivé à la même interprétation qu'il a encore un peu plus développée. Il considère les circonvolutions cérébrales et les sillons comme une accommodation réciproque à l'espace, de la matière grise et des fibres conductrices qui forment la substance blanche. Sans plis de l'écorce, il y aurait chez les grands animaux trop d'espace pour la substance blanche. Cette manière de considérer me paraît fertile.

On a cherché à maintes reprises la signification plus profonde

de cette extension de la surface de l'écorce dans ceci, que l'ali-

1 Ibid, p. 116.

2 Comparer les figures aux pages 115,119 et $_{\mathbf{6}} \mathbf{1 2 0}$, ibid, avec la table IV et fig. 3 de la table VII, chez Kükenthal_et Ziehen, Das Centralnervensystem der Cetaceen, Iéna, 1889.

3 Recherches sur la structure de la couche corticale des circonvolutions du cerveau. Paris, 1840

4 G. Jelgersma in Morphol. Jahrbuch. Bd. 15, 1889, p. 76, 77, et.autres. 
mentation doit se faire de la surface et qu'ainsi l'écorce ne peut pas bien se développer en épaisseur. Henle dit : toujours la forsurface des circonvolutions a pour but et pour suite d'agrandir la surface des hémisphères au profit de l'amenage du sang - et plus port avec la en circonvolutions est, en premier lieu, en rapdans une dencéphale. Car puisque le volume croit fonclion de la surface, proportion que la surface, pour que la augmente dans la consistant que dans l'amenage du sang, sion de la surface, c'est-à-dire son que le volume, l'exten. avec le volume, son plissement, doit s'accroître vantagé en comparaison un cerveau plus massif serait le désaavis. Dans l'afflux du san un petit cerveau ${ }^{1}$. Flower est du mème couche corticale réside, à c'est-à-dire dans la nutrition de la de plis. Dans la subst, a mon avis aussi, la cause dela formation veux central s'effectue un échan ou ganglionnaire du système nerrésulte que dans sa fonction elle dépe de matière très actif, d'où il l'afflux du sang. Tousles ganglions dend d'une manière excessive de seaux sanguins et le cerveau rions sont richement pourvus de vaissang chez l'homme, par quatre gat son sang, le cinquième de tout le la circulation amène aussi rapidementes artères. La suppression de tion. Les expériences de Mosso avectla suppression de leur fonctres appareils démontrent dans quelle pléthysmographe et d'auflux du sang augmente avec la folle importante proportion l'afmontre aussi que l'échange de matièren du cerveau. Mosso déla veille doit etre considérablement plère dans le cerveau pendant scles. Elle devient souvent si considérabande que dans les mudépasse celle du sang, et pendant peut diminuer au point de rester en dessouseil et la narcose elle consommation considérable de matièrsous de celle du sang. La Sur le ếparée par le Sur le terrain de l'anatomie de la substance grise du cerveau comparée la dépendance étroite par sa surface libre, se démontre l'afflux du sang ayant lieu chez tous les mammifères ellontre tout d'abord par le fait lieu grandeur du corps n'a sur cette épaissès la même épaisseur. La 1 Traité d'anatomi do l'hour 2 Die temperatur des Gehirnes, Neurologie.

ques et la temperature du cerveau. Leipzig, 1894. Aussi

(1892), p. 299 et La Peur, traduit Philos. transact. Les phénoménes psychi, Le London, vol. 183 nime. Kükenthal et Ziehen donnent comme épaisseur moyenne de l'écorce grise, mesurée dans les sillons du cerveau d'une baleine Hyperoodın rostratus), qui après durcissement dans l'alcool absolu pesait encore 2740 grammes, 2 à 3 millimètres ${ }^{1}$. Chez le bœuf, pour un poids de l'encéphale s'élevant à 350 grammes, je constate la moyenne de 2 millimètres, et chez le lapin sauvage dont l'encéphale pèse 9,7 grammes, la mème épaisseur moyenne de la substance corticale. Au cerveau de l'homme, dont le poids moyen est d'environ 1400 grammes, on trouve l'épaisseur de 2 à 3 millimètres. Cependant pour posséder, malgré cette épaisseur toujours à peu près égale, la même structure et en conséquence la $\mathrm{m}^{\hat{s}} \mathrm{me}$ fonction comme organe chez les grands comme chez les petits animaux apparentés et occupant le mème rang en organisation, le cerveau n'a pas seulement besoin d'un plus grand nombre de fibres conductrices, mais aussi d'un accroissement proportionnel de la quantité de l'écorce. Pour cela, il est trois possibilités: sans un épaississement considérable, le volume de l'écorce cérébrale peut augmenter :

10 Sans se plisser; par accroissement considérable du volume de la substance blanche, c'est-d̀-dire par allongement des fibres;

$2^{\circ} \mathrm{Par}$ le plissement seulement, sans augmentation de la substance blan he;

$3^{0}$ Tant par plissement de l'écorce que par augmentation de la substance blanche.

Dans le premier cas les fibres qui forment la substance blanche subissent, d'après la loi géométrique du rapport des surfaces avec les volumes un allongement consécutif considérable; dans le second cas l'allongement fait défaut, dans le dernier cas il est moins considérable.

Le premier mode d'agrandissement du volume de l'écorce cérébrale, qui ne change presque.pas en épaisseur, se rencontre exclusivement chez les petits animaux. Dès que la taille dépasse une certaine mesure, non identique pour toutes les ordres, la substance corticale se met en plis. De cette manière une perte superflue de matière est évitée ainsi qu'un agrandissement généralement désavantageux du crane. Certainement par le plissement de l'écorce le cerveau arrive à une épargne considérable de matière. La distance qui sépare les différentes parties de l'é-

1 Op. cit. p. 101. 
corce entre elles et des autres centres peut devenir relativement moindre, toutes les fibres qui arrivent des parties de l'écorce repliées en dedans peuvent devenir en proportion plus courtes. Dans de la surface libre. La il y a $2 / 3$ de la substance grise à l'intérieur obtenue, peut donc ètre dianche, ainsi doive diminuer, le nombre D'autres besoins de l'

fluence sur la mesure cérébrale et par augmentation laquelle par plissement de l'écorce che, c'est-à-dire par allongement volume de la subslance blantient l'augmentation dubres conductrices, s'obcorce peut se plisser moins ou plu cerveau. Selon ces besoins l'épour d'autres raisons l'agrand pue de coutume; si cerveau suit passivement, et l'écorce se plisse relativement fibres médullaires,

Il se trouve qu'en général le moins, et vice-versa.

grandeur dans telle mesure plissement n'augmente pas avec la médullaires deviendrait par que l'allongement relatif des fibres veau du tigre n'est pas pour là superflue. Par exemple le cervolution que celui du chat. On pent amment plus riche en circonqui ressortira surtout de faits a mettre comme certain, ce veaux des grands animaux antionner encore, que les cerriches en substance blanche que en général relativement plus rentés, mais seulement par allon des petits animaux appaaugmentation relative de leur nombement des fibres, point par cérébrale a donc moins de noids de substance chez les petits animaur chez les grands animaux

On peut arriver par une autre voie au mème groupe naturel. un rapport entre le poids du vere que Brandt et Snell à trouver du corps. Elle revient en résumé à la grandeur de la surface sances actuelles sur la structurée à ceci. D'après nos connais doit considérer l'encéphale de du système nerveux central, on formé des parties centrales chaque animal comme entièremen nexion les nerfs sensitifs et moteurcs reflexes, qui mettent en con ganisation plus ou moins élevér, lesquelles parties d'après l'orcompliquées. Chez des animaux de l'animal sont plus ou moins est le mème, le nombre des aux, dont le degré de l'organisation ments mares reflexes et ments nerveux n'augmentent nullement - parce que les élégré que la grandeur du corps - leur poids grandeur au même de- tivement en proportion du nombre des fibres nerveuses sensitives. Il se pourraitque chez des animaux de mème organisation, mais de grandeur différente, il faille évaluer le nombre des fibres sensitives d'après la grandeur tolale de la surface perceptive des sens, et celle-ci de son côté d'après la grandeur de la surface du corps. Si cela était, on aurait dans la surface du corps chez les animaux de même organisation, mais de grandeur différente, une mesure pour déterminer le poids de leur encéphale. Le chiffre obtenu de cette manière exigerait cependant de suile une cerrection de l'influence précitée, résultant de ce que l'écorce du cerveau n'accroit pas sensiblement en épaisseur. Mais aussi il paraîtrait qu'on ne peut absolument pas rapprocher directement la grandeur de la surface du corps de celle de la surface perceptive des sens et du nombre des fibres sensitives, même chez les animaux qui ne diffèrent que par la taille, mais qui du reste ont la mème organisation. Ce rapport est plus compliqué du fait qu'à mesure que le corps est plus petit, le nombre des fibres nerveuses sensitives augmente relativement. Une partie des surfaces perceptives des organes de sens devient relativement plus étendue, dans une autre parlie de ces surfaces les terminaisons des fibres nerveuses sensitives deviennent plus denses. Or c'est seulement dans le nombre de ces fibres qu'on a à chercher la mesure de l'encéphale chez les animaux de mème organiṣation.

De deux animaux d'organisation différente, mais de mème grandeur et avec des surfaces perceptives des sens à peu près de même étendue, et un nombre de fibres nerveuses sensitives à peu près égal, aura le cerveau le plus lourd celui chez le quel les parties centrales d'arcs reflexes en question, dont l'encéphale se compose, ont atteint la plus haule complexité. Cela découle de ce qu'un grand nombre de recherches sur divers terrains nous ont appris sur la structure du système nerveux. C'était certes là une vue lumineuse, comme dit Schopenhauer ${ }^{1}$, par laquelle Sömmering ${ }^{2}$ déjà, il y a plus d'un siècle, en vint à cette conclusion, qu'on ne doit pas évaluer le poids de l'encéphale d'après celui du corps entier, mais d'après l'épaisseur des nerfs.

Cette conclusion et l'analogue, à laquelle les connaissances

1 Parerga et Paralipomena. Leipzig. Edition Reclam., t. II, 393.

2 De basi encephali et originibus nervorum cranio egredientium libri. $V$ Göttingen, 1778, p. 17. 
actuelles sur la structure du système nerveux central me paraissent devoir conduire nécessairement, demandent à être précisées
et motivées.

Les recherches d'anatomie comparée et d'embryologie nous ont révélé que le système nerveux est formé primitivement d'unité se succédant sous forme de segments. On prités unités sensori-motrices. A la suite chaque partie du corps pénètrente fe fait les nerfs sensitifs de des mammifères près du point aussi dans la moelle épinière mandent les mouvements qui compar certaines fibres par certaines fibres sensitives sont renvoyées en premier lieu aux muscles de la mème partie. Cela prouve que certaines fibres nerveuses centripètes voisines - émanations de neurones sensitifs, avec certains neurones moteurs connexion dans la moelle épinière à des cellules musculaires déte voisins et ainsi appartiennent sensori-motrices ou arcs réflexes segments, sont en connexions primaires, appartenant à divers cellules d'association, et de par les longs prolongements des motrices plus parfaites manière des combinaisons Des coordinations

plus grande des mouveme plus compliquées et une efficacité des appareıls sensori-moteurs qui résultent d'une union semblable cés au crâne dans les organ qui ontleurs organes terminaux plaréflexes primaires du sens dont sens supérieurs, avec des arcs ainsi les muscles se trouvent le siège principal est la peau. Car, d'un sens.

combinée de plus sensori-moteurs secondaires, qui primaires se développent les arcs de l'écorce cérébrale. Les im qui ont leurs sommets dans les centres centripètes peuvent arriver aux conduites par voie des nerfs la moelle non seulement directemtres des nerfs centrifuges de diaire des cellules plus haut placées mais aussi par l'intermél'acte est devenu conscient. A mesures d'écorce cérébrale; par là un plus haut degré de développeme que le mammifère atteint sensori-moteurs secondaires de pluement, il s'est formé des arcs quoi l'on trouve que les faisceas en plus nombreux. C'est pourrelations les cellules efférentes (motramidals, qui mettent en les centres inférieurs du système lees) de l'écorcecérébraleavec proportionnellement plus larges nerveux, sont chez l'homme
Les centres des appareils sensori-inoteurs secondaires forment dans l'écorce du cerveau des zones dẽ projection, ainsi nommées: la zone tactile-motrice, la zone visuelle, la zone auditive et la zone olfactive.

La structure sensori-motrice primitive est dans les appareils secondaires des sens supérieurs très obscurcie par cela que leur partie centripète a gagné une plus grande importance en comparaison de la partie centrifuge, que ce n'est le cas avec les appareils sensori-moteurs du sens tactile. Mais que l'organisation primitive des zones cérébrales sensorielles est lamême que celle des appareils sensori-moteurs primaires cela est prouvé par là que la réaction motrice directe, qui suit l'excitation d'une de ces zones, s'effectue dans les muscles qui sont en relation avec l'organe du sens en question. Par excitation de la zone optique Ferrier vit se mouvoir chez les singes et les chiens les globes oculaires, et quelquefois la pupille se rétrécir; par excitation de la zone acoustique les oreilles se mouvoir. Toutefois, la masse musculaire qui a sa surface sensible principale dans la peau est en comparaison des muscles des autres surfaces sensorielles très grande et très différenciée. D'où il résulte qu'aussi sa localisation dans le cerveau est d'autant plus différenciée, que l'on a appris à connaître en premier lieu la réaction motrice de la zone en question et que l'on a qualifié jadis, à dater des expériences de Fritsch et Hitzig, celte zone comme « zone motrice ». H. Munk a ensuite démontré que la projection centrale de la périphérie sensitive et motrice s'y combinent.

Tant la périphérie motrice que la sensitive sont représentées dans les grands hémisphères d'une manière très localisée. A chaque point de la périphérie correspond un point du centre dans l'écorce grise du cerveau. Pour la périphérie motrice cela pouvait seulement ètre démontré, pour les raisons indiquées, de la zone tactile et sa partie motrice, qui embrasse presque tous les muscles du corps. Beevor et Horsley virent chez des singes se succéder dans l'écorce les centres pour l'épaule, le coude, le poignet, les doigts et le pouce, et aussi pour la hanche, le genou, le pied et les orteils, dans le mème ordre que ces parties se suivent à la périphérie dans les membres. Mème pour les fléchisseurs et les extenseurs ils constataient une localisation distincte.

Pour la périphérie sensorielle on a pu démontrer que, en premier lieu à chaque sens correspond une certaine partie de la surface du cerveau, qui est analogue chez différents animaux 
examinés à ce sujet. Mais plus loin on a aussi prouvé que les parla nuque et de membres antérieurs et postérieurs, du tronc, de zone sensitive la tète ont leur localisation représentée dans la a pu démontrer que chaque pointus important c'est que H. Munk un point déterminé de l'éco point de la rétine est en relation avec On comprend donc asémé cérébrale.

jection correspondante doit aunt que l'étendue de la zone de proélevée de chaque organe de augmenter avec la différenciation plus surface de perceplion que sens, et pourquoi celle d'une si petite inférieure à celle du sens la rétine n'a pas une étendue beaucoup corps et qui a en outre tactile, qui s'étend à toute la surface du tantes d'extension. Suivant les à l'intérieur des surfaces imporenviron un demi milliont les calculs de Stilling, chez l'homme dans la moelle épinière, de fibres nerveuses sensitives entrent le nerf optique est composé le nombre de fibres nerveuses, dont même nombre. C'est dans

tion que réside le pouvoir de l'aptituent les zones de projecmotrice acquiert ainsi, d'ar de l'aperception. La zone tactiloet de perfectionner les mouvement, la signification de régler encore plus parfaite. Du moiments, de rendre leur efficacité riences faites chez les singes et les cela paraît résulter des expépathologiques chez l'homme tes chiens ainsi que des observations une certaine partie de la zone tarsque chez l'homme, par lésion, convolutions centrales) est mise hoctilo-motrice (le centre des cirréflexes et automatiques du bras hors de fonction, les mouvements vement volontaire est devenu imeuvent persister, mais le moutaire ne mouvement; s'effectuer que par le more. Le mouvement volonni celles mouvement partent de la peau, atteint plus l'écorce cérébrale, aucun mouvemext dans les tendons celles qui proviennent du les appareils sent volontaire ne se produit ligaments, et par la ment liée à sensori-moteurs secondaduit plus. Donc aussi, dans Sur les à motilité.

enfin, entre les différentes zones sens secondaires se développent
fères supérieurs, appareils d'a comme arcs réflersorielles, chez les mammiappareils d'association tertiaires, les de l'ordre le plus élevé, les
Flechsig. D'après Flechsig ils manquent complètement chez les rongeurs, et sont encore peu développés chez les carnivores, mais atteignent chez les singes la même étendue que les centres sensoriels; chez l'homme cependant l'écorce grise est centres sensoriels; chez l'homme cepend '. Par le fait qu'ainsi les perceptions de divers sens peuvent s'associer, le cerveau obtient enfin' comme organe sa plus haute signification fonctionnelle.

Ces connaissances acquises sur la structure et les fonctions de la partie la plus différenciée et la plus étendue de l'encéphale, le cerveau, ainsi que de la partie la plus inférieure et la pius primitive, la moelle épinière, rendent le fait probable que les parties de l'encéphale dont la structure et la fonction sont encore moins connues, sont aussi construites suivant le même type. Nous pouvons considérer l'encéphale comme formé d'unilés, plus complexes chez les animaux supérieurs, moins complexes chez ceux qui se trouvent à un degré plus bas de développement phylogénétique, plus nombreuses chez les animaux de grande taille moins nombreuses chez les animaux de petite dimension. Nous avons appris à connaître la fonction comme intimement liée à la avons appris a conane central, gagnant en perfection à mesure que l'organe devient plus complexe, de sorte que nous poussi une structure identique là où nous observons une très grande analogie, même dans les fines nuances de la fonction. Les animaux dont les fonctions psychiques présentent une si grande analogie comme cela se voit, par exemple, dans la famille des chats, doivent aussi s'accorder à ce point dans la structure intime de leur système nerveux central, que nous pouvons, pour les considérations, qui forment le sujet de cet article, les considérer comme équivalents.

Chez les animaux qui sont de la même taille, la quantité de l'encéphale dépend donc du degré de la complexité qu'il a atteint. La masse et le poids de l'encéphale augmentent dans une proportion égale à celle de sa complexité, car continuellement des arcs reflexes secondaires sont superposés aux primaires et enfin des tertiaires aux secondaires. Ce qui par conséquent augmente c'est la céphalisation du système nerveux central, tant en qualité qu'en quantité. Toutefois nous ne pouvons encore exprimer en quelque sorte en chiffres que la céphalisation quantitative.

1 p. Flechsig, Gehirn und Seele, 2. Ausgahe, Leipzig 1896, p. 23 ot 84. T. VIII ( $4^{0}$ SÉRIE). 
On avait d'abord tenté cela par la comparaison du poids ou de la largeur de l'encéphale avec celui ou celle de la moelle épinière. L'homme, dont le poids de la moelle épinière n'atteint pas celui de cet organe chez les grands mammifères, n'est pourtant dépassé dans le poids de l'encéphale que par quelques-uns des plus grands. L'encéphale pèse chez l'homme 50 fois, chez le chien et le chat 3 à 4 fois, chez le cheval 2 fois $1 / 2$ et chez le lapin 2 fois autant que la moelle épinière ${ }^{1}$. La moelle allongée mesure à sa base, chez l'homme seulement $1 / 7$, chez le cheval, le bœuf, le mouton, le cerf, geur de l'encénien, le chat et le lapin $1 / 3$ ou peu moins de la lar-

Ensuite, comme nous l'avons déjà fait remarquer, Sömmering a pensé le premier à mesurer la céphalisation duer, Sömme nerveux le nar la grosseur relative des nerfs, en d'autres termes, par l'encéphe relatif des fibres nerveuses qui sont en relation avec seur des font presque chez les différents animaux, les données voulues sorte à jue tolalement défaut. On pourrait arriver en quelque musculaires d'anim rapports par la comparaison des éléments même raille différente mais appartenant à un ne sont pourvues La plupart des fibres musculaires striées comme chez des que d'une seule fibre nerveuse motrice. Or férente, les masses demblables, mais de grandeur très difport que le poids dues muscles analogues sont dans le même rapleurs éléments muscorps, on pourrait juger le nombre relatif de d'après le musculaires, ainsi que desfibres nerveuses motrices, fibres musculaires, donc d'après la longueur et l'épaisseur des acquis, à maires chez les animaux comparés. Les rares faits fibres musculaires que le nombre des femblent en harmonie avec cette supposition, mesure moindre le poids du corps. la masse des muscles, et augmente dans une tules musculaires par conséquent que animaux congénères. ${ }^{1}$ Bischoff, Das Hirngewicht des

of the brain, p. 191. Ranke, des Menschen, p.37 N 10,1895 Table I. Ranke, Corresp. Blatt, p. 37. Donaldson, The growth

2 Cuvier,

Cuvier, Leçons d'anatomie comparée, $9^{\circ}$ lecon. striés ont 3 à 24 millimètres de long et chez le lapin 60 à 75 millimètres, chez le mouton 20 à 35 millimètres et chez le bœuf 80 à 100 millimètres ${ }^{1}$. L'épaisseur varie chez la souris de 0.0038 à 0.0798 millimètres ${ }^{2}$, tandis que les fibres musculaires de l'homme, avec une longueur de 53 à 98 millimètres, ont une épaisseur de 0.011 de 0.072 millimètres ${ }^{3}$. Il se pourrait donc bien que le nombre des fibres nerveuses motrices augmente avec la section, non avec le volume du muscle, comme déjà Brandt, se basant sur la thèse, prouvée insoutenable, de Kölliker, que les fibres musculaires striées sont toujours aussi longues que le muscle tout entier, l'avait supposé

Pour deux espèces d'animaux intimement apparentés et de même forme, mais de grandeur très différente, on peut à peu près évaluer le nombre relatif de leur's fibres nerveuses sensitives d'après la grandeur des surfaces sensorielles, et celles-ci doivent en quelque sorte ètre jugées d'après la grandeur de la surface du corps, sans cependant, comme il est déjà dit, lui être proportionnelles. Qu'il puisse ètre question d'une surface sensorielle, d'une différenciation non égale chez les différents animaux, cela s'entend facilement pour la peau; mais les terminaisons périphériques des nerfs sensitifs internes sont également ètendues en surfaces, dans les muqueuses, les séreuses, les aponévroses, les ligaments. Les nerfs olfactifs et auditifs sont également déployés dans les muqueuses. G'est dans les membranes du Labyrinthe que s'étendent les appareils terminaux périphériques du nerf auditif. La rétine est une membrane.

En comparant de plus près les surfaces sensorielles avec la surface générale, l'on a depuis longtemps trouvé que pour la surface sensorielle de l'œil, il n'existe certainement pas un rapport proportionné à la surface générale. Haller savait déjà que les petits animaux ont proportionnellement de plus grands yeux que les grands; cela se remarque le mieux en comparant des animaux intimement apparentés, comme le chat, le lynx et le lion ". C'est à ce point que la grandeur linéaire des

1 R. Mayeda in Zeitschrift für Biologie. Bd. 27, 1890, p. 119.

2 W Fèlix, Die Länge der Muskelfaser bei dem Menschen und einigen Säuge thieren, Würzburg. 1887 , p. 281.

3 Vierordt, Daten und Tabellen für Mediciner 2. Aufl. Jena, 1893, p. 63.

4 R. Leuckart, Organologie des Auges. Handbuch der gesammtem Angenheilkunde von Gräfe und Sämisch Bd. II, cap. vII, p. 181. 
images sur la rétine est chez le lion seulement de 1.67 fois, et la surface de ces images 2.8 fois aussi grande que chez le chat ${ }^{1}$. Le poids du corps du lion est à peu près 36 fois celui du chat, dont on peut déduire que la surface du corps du lion est environ egale à 11 fois celle du chat. On sait de plus que les éléments pour la perception de la forme, les batonnets de la rétine, ont chez la plupart des mammifères la même grosseur, d'où il s'en suit que par millimètre carré, sur la mème surface de membrane rétinienne il y en a toujours à peu près le mème nombre ${ }^{2}$, chez le chat comme
chez le lion.

Si chez ces animaux la surface de la membrane rétinienne était en proportion de la surface du corps, le chat verrait un mème objet aussi clairement à la distance de $1 \mathrm{~m}$. que le lion le voit à $V 11=3.3 \mathrm{~m}$. Mais parce que chez le chat la réline est relativement environ 4 fois plus grande, l'o chat la rétine est relativepeut déjà ètre égale à grande, l'acuité visuelle de cet animal plus rapprochés de luique du lion, si les objets sont de 1.67 fois sement de son eil, luique du lion. Le chat voit, par cet agrandistance de celle quil les objels aussi clairement à une double disn'a qu'à les appros verrait sans agrandissement de son œil, ou

En règle générale, de la moitié.

ont des yeux relativemez tous les vertébrés, les petites espèces parentées. Les images de plus grands que les grandes espèces aptera sibbaldi) sont linéai la réline de la baleine géante Balaenopcelles de la rétine du les dimensions linéa marsouin (Phoraena communis), tandis que 10 à 20 pour 1. Les de leurs corps sont en proportion de pêcheur sont à pein images sur la rétine de l'orfraie ou aigle la crécerelle, tandis que laussi longues et larges que celles de fraie sont 3 i 4 fois que les dimensions linéaires du corps de l'ormert a lrouvé chez le grandes que celles de la crécerelle ${ }^{3}$. Emet le lapin que, sans cheval, le bœuf, le mouton, le porc, le chat, espèce ont relativement les les plus petits animaux de la mème ${ }^{1} \mathrm{~L}$. Mathi

optischen Bau des Die neueren Fortschrite

R. Leuck des Auges der Wirbeltieritte in unserer Kenninis von dem ${ }^{3}$ Leuckart, Ibid. p. 249. est dèsigne tt Matthiessen.

le genre Delphirsouin (Phocaena - Par Delphinus communis, sans doute $1 Z$ eitschinus.

Wiesbaden, 1886.
.

Il est également presque certain que, en général, chez les petits animaux la peau contient par unité de surface considérablement plus de terminaisons périphériques de fibres nerveuses sensitives que chez des animaux plus grands et qu'ainsi la surface sensorielle du sens tactile est plus serrée chez les petits animaux. L'animal veut-il dans ses rapports avec le monde extéricur employer divers sens combinés, cela ne peut pas être autrement; un sens ne doit pas rester en arrière en acuité de sensation de l'autre. Des objets, rapprochés de l'œil à ce point qu'ils peuvent venir en contact avec le corps, le chat observera, a vec son œil relativement plus grand des détails linéaires qui ont sculement la moitié des dimensions de ceux que le lion peut dans ce cas observer. Le chat a à faire, aussi bien de près que de loin, avec des objets plus petits que le lion, qu'il ne doit pas moins bien pouvoir palper que les voir. Mais l'ouie aussi doit gagner en acuité, pour rendre l'accord avec les autres sens possible; par agrandissement de la surface sensorielle dans l'oreille, les ondes sonores trouvent une face d'appui plus étendue et des sons moins intenses peuvent être sentis de cette manière. Par extension de la muqueuse olfactive, une plus grande quantité de gaz et de vapeurs peuvenl venir en mème temps en contact avec elle. Mais concernant l'agrandissement relatif de ces deux derniers sens il n'existe pas de données.

En tout cas il se trouve que la zone optique de l'écorce cérébrale n'est pas plus développée chez les petits animaux que chez les grands, mais tout à fait dans la mème proportion que la zone tactile et la zone auditive; comme par exemple chez le chat commun, le chat jaune des forèls de Sumatra (Felis temmincki) et le tigre, animaux apparentés mais formant une série quant à la grandeur du corps.

Tout de mème par celle influence généralement chez les petits animaux l'encéphales'agrandit d'une manière importante. On peut démontrer la constance de ce facteur, par lequel, chez des animaux de même groupe nalurel, le poids de l'encéphale augmente en beaucoup plus faible raison que la surface du corps, plus loin encore. Que les organes des sens chez les petits animaux sont faits pour apercevoir à des distances relativement plus grandes et en mème temps pour des objets plus petits, que ces petits animaux ont en comparaison de leur taille un champ d'observation plusétendu que les animaux de grande taille, appartenants au 
même groupe naturel, cela est indubitablement lié à ce qu'ils ont besoind'un plus grand champ d'activité pour pouvoir satisfaire aux besoins de leur échange de matière, qui est tant plus forte. Chez le chat, celle-ci est par unité de surface de corps environ aussi considérable que chez le lion. Comme le grand animal pèse 36 fois autant que le petit animal, mais présente seulement une 36 fois autant 11 fois aussi grande, l'échange de matière chez le chat est 3.3 aussi considérable par unité de poids que chez le chat est 3.3 tité de travail musculaire que l' poids que chez le lion. La quanmême proportion et suivant animal doit faire est calculée dans la leurs terminaisons ralement il semble par dansl'encéphale, sont disposés. Généde la densité des terminar conséquent admissible que le facteur sation chez les grands et los sensitives dans les surfaces de senturel présente une valeur constante. eur constante.

Les dimensions dest-être motivée de la manière suivante. sont en proportion de celles, auxquels chaque animal a à faire, donc en moyennne 3.3 fois de son corps. Les objets du lion sont grands en surface, 36 fois plus longs et plus larges, 11 fois plus animaux touchent main plus lourd que ceux du chat. Si les deux patte, il se trouve à une diant leur objet, par exemple avec Ia lion que de celui du chat distance 3.3 fois plus grande de l'œil du grande, l'intensité de l'imais il a aussi une surface 3.3 fois plus deux animaux, dont les yeux rétinienne est donc égale chez les autant de fibres nerveuses sont uniformes. Chez le lion 2.8 fois image que chez le chat. En optiques sont influencées par cette rence qualitative dans les nerfs apparentés, il faudra, pour que chez ces animaux intimement tions reçues d'un mème objet dans lême rapport entre. les incitaservé - comme il convient dans les différents sens reste conrentés - que 2.8 fois autant chez des animaux intimement appale chat. Mais comme la surfacerfs tactiles soient influencés chez grande que celle du chat, la dactile du lion est 11 fois plus de la surface tactile peut la densité des terminaisons nerveuses du chat. Et par ce que, généralemviron que $\frac{2.8}{11}=\frac{1}{4}$ de celle plus grandes intimés ont les yeux plus es esèces plus petites doivent aussi, intimement apparentées, les tonds que des espèces compacte dans la peau.
listribuer d'une manière plus
Cela est d'accord avec la manière dont le système musculaire fonctionne. La force musculaire chez des animaux intimement apparentés est, en rapport avec le poids du corps, d'autant plus petite que l'animal est plus grand, sans que l'on doive supposer quelque différence qualitative dans les muscles. Elle est tout à fait indépendante de la longueur, mais au contraire, comme l'on sait, proportionnée à la section du muscle, donc chez le lion 11 fois aussi grande que chez le chat. Notons que cette force $\mathbf{1 1}$ fois plus grande a à déplacer un poids 36 fois plus considérable, ce qui provient de ce que les parties du corps du lion sont d'autant plus lourdes, ainsi que les objets auxquels il a à faire. Par suite de cette plus grande charge, le muscle ne peut se contracter que plus lentement. D'où les mouvements chez les grands animaux sont plus lents que chez les petits. C'est un fait à observer généralement que les petits animaux sont plus agiles dans leurs mouvements, que dans la locomotion leurs membres se remuent plus lestement que chez les grands. Les pas du tigre, marchant à son aise, sont beaucoup moins agiles que ceux du chat, lorsque celui-ci se déplace de la mème manière. Si les membres de la souris ne faisaient, dans un temps égal, plus de mouvements que ceux du cheval, sa locomotion ressemblerait, dit Plateau, à celle d'une tortue. C'est à cette faculté que les petits animaux doivent de ne pas trop être dépassés en vitesse absolue de locomotion par les grands, que par exemple le renard et le lièvre ne courent pas moins vite que le cheval, et que des chiens peuvent attrapper un cerf. Tous les petits animaux répètent leurs mouvements dans un rythme plus rapide que les animaux plus grands, et l'influence détavorable de leurs faibles dimensions corporelles, sur la vitesse absolue de la lucomotion, est en grande partie compensée de cette manière.

C'est ainsi que l'observation directe nous l'apprend et l'on peut démontrer, que la raison de ce phénomène admirable setrouve dans de simples rapports mathématiques. Si, de deux animaux apparentés et de forme très semblable, l'un grand et l'autre petit, $L$ et $l$ sont les dimensions linéaires, $M$ et $m$ les masses, $V$ et $v$ les vitesses de la locomotion (c'est-à-dire les distances parcourues par les parties analogues des corps dans l'unité de temps), $\boldsymbol{F}$ et $f$ les forces musculaires, nous avons

$$
\frac{F \cdot L}{f \cdot l}=\frac{M \cdot V^{\mathrm{e}}}{m \cdot v^{2}}
$$


Les forces musculaires se rapportent chez des animaux de même fions dez intimement apparentés assez exactement comme les sections dez muscles, et les masses comme les sec aussi mettre

$$
\text { D'où il suit } \quad \begin{aligned}
\frac{L^{2} \cdot L}{l^{2} \cdot l} & =\frac{L^{3} \cdot V^{2}}{l^{3} \cdot v^{2}} \\
V & =v
\end{aligned}
$$

Le petit animal parcourt d

blance parfaite, et parcourt donc (dans la supposition de ressemtement comme les en admettant que les forces se rapportent exacmin dans le même têns dans les muscles analogues) le même chementaires des parties que le grand animal. Les mouvements éléquence chez les animaurrespondantes du corps, doivent en conséles muscles correspondax plus petits se répéter plus rapidement, et Pour chaque mouvements contracler dans un temps plus court. continue ou naturelle d'est-à-dire pour chaque contraction (quand on naturelle d'une fibre musculaire, un que contraction ne dispose pas d'une impune différence qualitative dans les nerfs tit animal. Dans le impulsion nerveuse plus forte qu'un plus peexciter la contraction cas une dose égale doit affluer aussi pour lières, dont secompon, et par conséquent les contractions singurel, devrontètreégale la conl raction conlinue du mouvement natutinue soit pourtant de nombreuses. Pour quela conement natufibres pourtant de plus courte for musculaires doivent ètre petit animal, ses ces fibres et des fibres nerveuses petites et le nombre relatif de rapport entre les dimensions line ètre $\lambda$. fois plus grand, si $\lambda$ est le fibres musculaires et nerveins linéaires des animaux. Plus il y a de plus nombreuses sont les expl, plus il y a de points d'excitation, substance du muscle pendantosions d'énergie moléculaire dans la de est la contraction due même temps, et d'autant plus ragie du muscle à l'état potentiel. Chez l'animal plus grand l'énernerveuse, doit se dévelo potentiel, mise en liberté par l'excitation parce que le seder plus lentement. grandes, et crimposi lume, moins qu'il reçit par consí de fibres musculaires plus tant, dan fibres nerveuses quent, en proportion de son voalors plus de fibre musculaire l'onde de contraction parfaut consio temps pour la pare, du point d'innervation, exige actuellement le système nerveux sa masse. S'il semble d'arcs réflexes, il doit exister, chez les animaux organisés de la même manière, le mème rapport entre le nombre des fibres nerveuses motrices et sensitives. La surface cutanée du plus grand animal est $\lambda^{2}$ fois, mais la dimension linéaire du corps n'est que 1. fois plus urande que chez l'animal plis petit, par conséquent, les fibres nerveuses tactiles doivent être chez le plus grand animal considérablement moins nombreuses, par unité de surface, que chez le plus petit.

Ainsi l'intensité de l'échange de matière est donc liée avec le poids du cerveau, mais seulement, indirectement et d'une toute autre manière que se le figuraient Brandt et Snell.

Ce facteur de la densité de la surface sensorielle, lequel rend l'encéphale relativement plus grand chez les petits animaux, aussi bien que le facleur agissant en sens invers:, qui s'écoule de l'épaisseur égale de l'écorce cérébrale, sont à considérer en cas comme fonctions mathématiques du poids du corps. En faisant abstraction pour un moment des deux facteurs, ainsi que d'autres influences, tels que la grandeur plus ou moins inégale des cellules ganglionaires chez des animaux intimement apparentés, mais de grandeurs différentes, nous arrivons à formuler semblable à Snell le problème qui fait le sujet de cet article de la simple manière mathématique suivante.

De deux animaux d'organisation égale et de mème forme, $a$ et $A$, dont les poids de leur corps $s$ et $S$ sont connus, les surfaces se rapportent l'une à l'autre comme ${ }^{3} V \overline{\mathrm{s}^{2}}:{ }^{3} \sqrt{\mathrm{S}^{2}}$; car, vu que les animaux comparés sont aussi à peu près conformes dans les parties internes du corps, on peut, sans faire une faute appréciable, établir les volumes en proportion des poids.

Admet-on que le poids du cerveau, chez ces animaux d'organisation également élevée et de forme identique, augmente proportionnellement avec la surface du corps, les poids de leurs encéphales doivent aussi être l'un à l'autre comme ${ }^{3} \sqrt{\mathrm{s}^{2}}:{ }^{3} \sqrt{\mathbf{S}^{2}}$, donc comme les racines cubiyues des carris des poids des corps. Au lieu de ${ }^{3} V \mathrm{~s}^{2}$ on peut aussi écrire $s^{2 / 3}$ ou bien $s^{06666 \ldots}$.

Suppose-t-on que la quantité de l'encéphale soit en proportion comme de la surface du corps, $0.6666 .$. serait donc le nombre qui, animaux posant, doit ètre ajouté aux poids de deux ou plusieurs le rapport du poimés par une certaine unité de poids, pour donner respondant à « l'ex de leurs encéphales. Qualifions ce nombre (cora "l'exposant somatique ) de Snell), l'exposant de re- 
lation, puisqu'il aide à exprimer l'étendue des relations avec le monde extérieur.

Voudrait-on comparer les poids de l'encéphale d'animaux d'organisation d'un degré différent, la valeur trouvée $\mathrm{s}^{0.6666 \ldots}$ devrait encore être multipliée pour chaque animal par un facteur qui exprime le degré d'organisation de l'encéphale, la quantité de celui-ci par unité de surface du corps, c'est-à-dire la céphalisation relative de l'animal en céphalisation certaine mesure au facto en facteur de céphalisation de Snell, nous l'appellerons l'encéphale d'un animal, dont le poids de c. $\mathrm{s}^{0.6666 \ldots}$. Le chiffre oberait égal à gnification relative, il donne telle manière a seulement une sil'encéphale chez les animauxe seulement la quantité relative de

De deux animaux dor comparés.

le poids des corps sont $s$ et $s$, égale et de mème forme, dont l'un à l'autre en proportion $\mathrm{S}^{0.6666 . .}$ : S 0.6666. .

Snell pensait

plus grande valeur que 0 . 666 sant somatique ) devait avoir une que suivant lui le volume du placée entre ce nombre et 1, parce une influence sur la grandeur corps, aussi bien que sa surface, fluence, dont j'ai parlé plus haut,encéphale. Cependant par l'inment des organes de sens entre de la différence en développel'exposant devra avoir au contraires grands et les petits animaux Cela ressortira des calculs suivants que chez les animaux qui diffèrent qu'il soit dejà remarqué ici, pour le reste comparables, toujours par la taille, mais par unité de surface du corps, plurs les plus petils présentent, le siamang $\quad 1.27$ autant d'encéphale que les grands : l'antilope pygmée 1.37 que l'orang-outan.

le chat $\quad 1.51$ le beisa

la souris $\quad 1.40$ - le lion.

En tout cas la quantité de l'encép le rat brun ${ }^{1}$ quement égaux, mais différents de certaine manière en rapport avec la grandeur corporelle, est d'une peut exprimer par le poids. De quelle mandeur du corps, que l'on lation à chercher nous l'apprendra.

1 Ces nombres sont calculés d'après la formule $c=\frac{\theta}{\mathrm{s} 0.66,}$
Pour trouver l'exposant de relation il est nécessaire de connaître de deux animaux adultes, semblables dans le degré de céphalisation de leur système nerveux central et de mème forme, le poids du corps et le poids de l'encéphale. Soit $e$ (encephalon) le poids de l'encéphale, $s$ (soma) le poids du corps du plus petit, et $E$ le poids de l'encéphale, $S$ le poids du corps du plus grand, $r$ l'exposant de relation, et $c$ pour tous deux le même facteur de céphalisation. On a alors les rapports suivants :

$$
\begin{aligned}
E: e & =c \cdot S^{\mathbf{r}}: c: s^{\mathbf{r}} \\
E: e & =S^{\mathbf{r}}: s^{\mathfrak{r}} \\
\left(\frac{S}{s}\right)^{\mathbf{r}} & =\frac{E}{e} \\
r(\log S-\log s) & =\log E-\log e \\
r & =\frac{\log E-\log e}{\log S-\log s}
\end{aligned}
$$

Pour l'exposant de relation $r$ on devra trouver, par le développement plus considérable et la densité plus grande de la surface sensorielle chez les petits animaux, ainsi qu'il est mentionné plus haut, en général une valeur en-dessous de 0.6666 .. . Le facteur qui résulte de la persistance de l'état mince de l'écorce cérébrale, agit cependant dans une forte mesure contre ce facteur de la densite de la surface sensorielle. La surface sensorielle du chat est probablement environ quatre fois aussi dense que celle du lion, ce qui n'empèche pas que le chat n'a en proportion de la surface de son corps qu'une fois et demi autant d'encéphale que le lion. Il y a donc une compensation pour l'encéphale du plus grand aninimal, par laquelle l'influence de son nombre relativement moindre des unités senso-motrices, est en partie effacée. Cette compensation ne peut-être trouvée que dans l'allongement des voies de conduction (et l'épaississement de leurs gaines médullaires) qui composent la substance blanche du cerveau. Il me semble qu'on nepuisse expliquer d'aucune autre manière comment, malgrél'augmentation beaucoup plus considérable du nombre relatif des éléments sensitifs du chat, le poids de son encéphale ne peut croltre qu'une fois et demi.

Seulement pour des mammifères chez lesquels les deux influences sont compensées à peu près l'une par l'autre, on devra trouver l'exposant de relation environ égal à $0.6666 \ldots$; pour la plupart des mammifères, on devra trouver une valeur inférieure 
à ce nombre. Mais comme les deux facleurs en question, agissant en sens contraire, dépendent également de la grandeur du corps, les chiffres trouvés par comparaison d'animaux semblables de chaque groupe doivent être à peu près identiques, si toutefois les idées sur la structure du système nerveux suivies dans ce travail sont justes.

On peut, avec l'aide des chiffres communiqués par Weber et en partie empruntés à d'autres, et par quelques nouvelles déterminations de poids, prouver d'une manière frappante la justesse de actuelles sur le syslèment comme de soi de nos connaissances Parmi lur le système nerveux central.

Parmi les séries des pesées communiquées par Weber, j'ai choisi attention à ce qu'elleces de différents groupes, en faisant bien forme du corps qu'elle suient autant que possible semblables en rentes en taille. Par une différion de l'encéphale, mais très diffécorps, des variations individerence considérable dans le poids du qui, pour l'espèce en question, peurent déviations des chiffres normales, ont moins d'inflon, peuvent ètre considérées comme Les animaux comparés doivent sur les résultats de la comparaison. représenter la taille et le poids nortout être entièrement adultes et reste, je ne me suis laissé condormaux pour leur espèce. Pour le ment 12 espèces ont été trouvé par rien dans ce choix. Seuleplupart des animaux énumérés aptes pour la comparaison. La le poids du corps qui, pour l'espent pas adultes, ou n'ont pas normal, d'après des indications tions personnelles. Aussi, dans chaques ailleurs ou des observarapprochés on ne peut que rarement groupe d'animaux très comparaison. Ces 12 espèces (table I), rener des couples pour la possible et par conséquent l'exposant rendaient 7 comparaisons calculé 7 fois (table II). C'est certes de relation $r$ pouvait ètre confirmation de la justesse de nos une surprenante el belle tion du système nerveux, que, chac idées actuelles sur l'organisarentes conduisaient au résultaque fois, des données si difféces idées. Toujours la valeur qui pouvait ê're attendu d'après rieure, mais pas trop, à 0.6666 . C'est donc vraiment l'étendu.

rielle qui détermine la quantité de l'a densité de la surface sensod'organisation égale.
TABLE I 1).

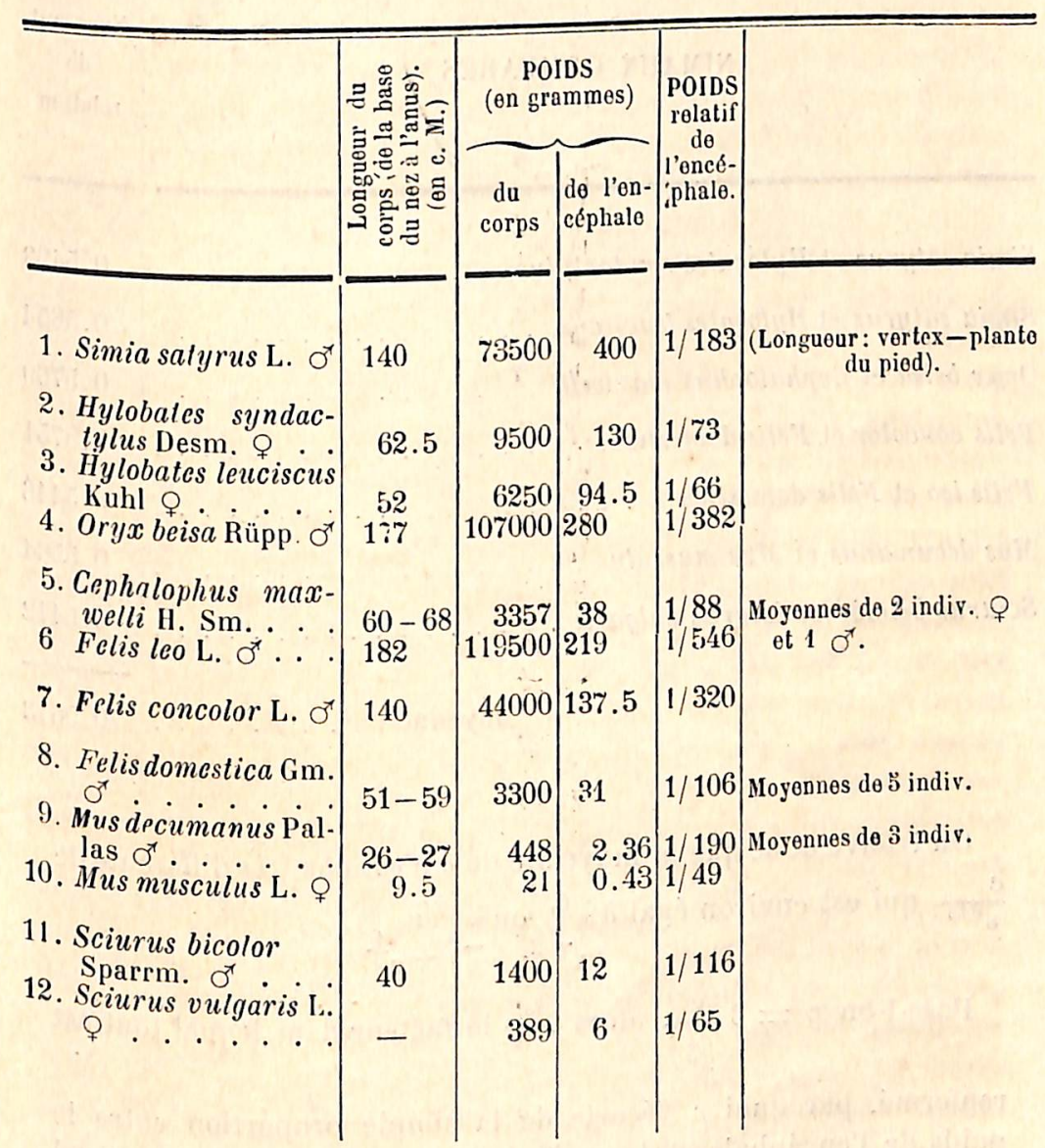

${ }^{1}$ Toutes ces donnèes niker el Boulart in $\mathrm{n}^{\circ}$ 11, d'après Kohlbruvge in Natuurk. Tijdschr. v. Ned.-Indië. Deel 55 1896, p. 31. 
TABLE II.

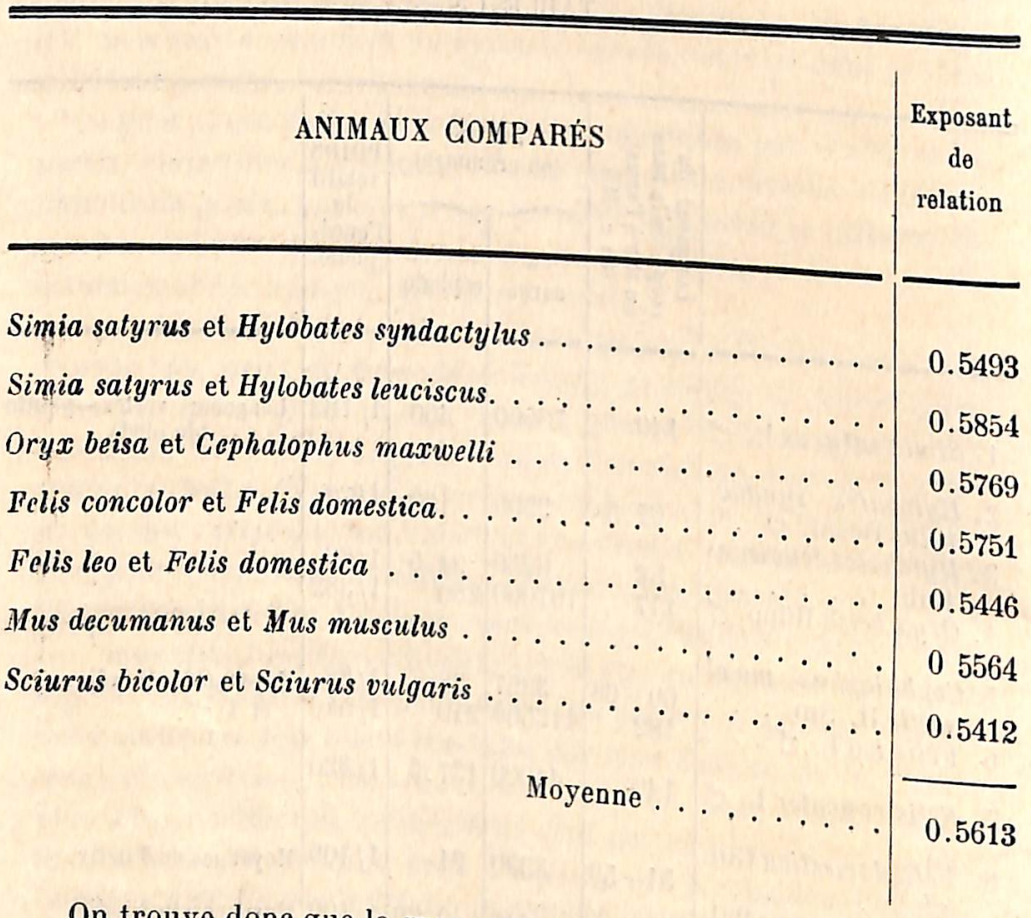

On trouve donc que la moyenne de $c$ n'est pas très différente de $\frac{e}{s^{0, B b}}$, qui est environ égal à $\frac{e}{s^{\frac{2}{3}}}$ ou $=\frac{e}{s^{3}-s^{\frac{1}{9}}}$

Pose-t-on $c=\delta \frac{e}{s^{\frac{2}{3}}}$, alors $\delta$ - le facteur dans lequel tout est renfermé, par quoi $c$ s'écarte de la simpl poids de l'encéphale et la surface du simple proportion entre le à $\mathrm{s}^{\frac{1}{9}}$, c'est-à-dire proportionnel avec lu corps - est environ égal dimension linéaire (du radius) du corps racine cubique de la Chez quelques animaux inférieurems de l'animal.

fluence des circonvolutions sur le poids organisés et petits, l'inpas. Ainsi, le cerveau chez les plus grandes dencéphale n'existe en Europe est aussi lisse que chez les plus tion relative de la matière blanche chez petites. L'augmenta- est donc plus considérable que dans les cerveaux oủ il y a plissement de l'écorce. Quoique les plus grandes espèces soient encore très petiles, elles atteignent cependant plus que quatre fois le poids du corps des plus petites espèces. Tous se trouvent à un très bas degré d'organisation de l'encéphale, et sont dans chaque rapport très rapprochées entre eux. Par là qu'ils ne s'écartent pas trop par la grandeur du corps, mais surtout par le très peu de développement de leur sens visuel, le facteur de la différence de densité et d'étendue de la surface sensorielle est plus petit que d'ordinaire. Leur sens principal est le sens tactile, dans le développement duquel ils paraissent dépasser tous les autres mammifères. Ils l'emploient pourtant d'une manière toute particulière, non comme sens du toucher proprement dit, et l'on peut admettre que dans le développement relatif de ce sens chez les différentes espèces, qui se ressemblent beaucoup dans leur mạière de vivre, dont la forme du corps est très semblable, et qui sont toutes petites, il n'existe pas de différence notable. Les animaux ayant servi pour la comparaison ont été pris dans les carrières souterraines de la Montagne de Saint-Pierre, près Maastricht, les uns près des autres dans un demi sommeil hivernal, vers la fin du mois de décembre 1896 , et lués bientôt après, cependantils ne purentêtre examinés que 12 heures plus tard, mais entre-temps ils furent conservés dans une température de $0^{\circ}$ à $2^{\circ} \mathrm{C}$. Chez aucun d'entre eux l'intestin ne paraissait contenir de restes de nourriture. Quatre animaux, bien adultes d'après les dents et les extrémités des os longs, furent choisis, l'état de nutrition de leur corps, la quantité relative de leur graisse paraissant très bien d'accord. A cause de la petite dimension des encéphales, la détermination du poids demandait des précautions particulières. Ils furent extraits soigneusement du crâne dans une atmosphère saturée de vapeur d'eau, aussi vite que possible, et séparés, tous de la même manière, de la moelle épinière près du grand trou occipital. Après enlèvement de la dure-mère chaque encéphale fut de suite mis entre deux verres de montre, et pesés sur une bonne balance à milligrammes. Des déterminations du poids-contrôle montraient, que mème à découvert et pendant un temps beaucoup plus long que n'avait duré l'extraction du crâne, aucune perte sensible d'humidité n'avait lieu dans la chambre saturée de vapeur d'eau. Des mesures prises des animaux employés, est donné seulement ici la longueur de l'avant-bras; la longueur du corps, la largeur 
des ailes étendues, elc., sont chez d'aussi petits animaux des mesures peu certaines.

TABLE III.

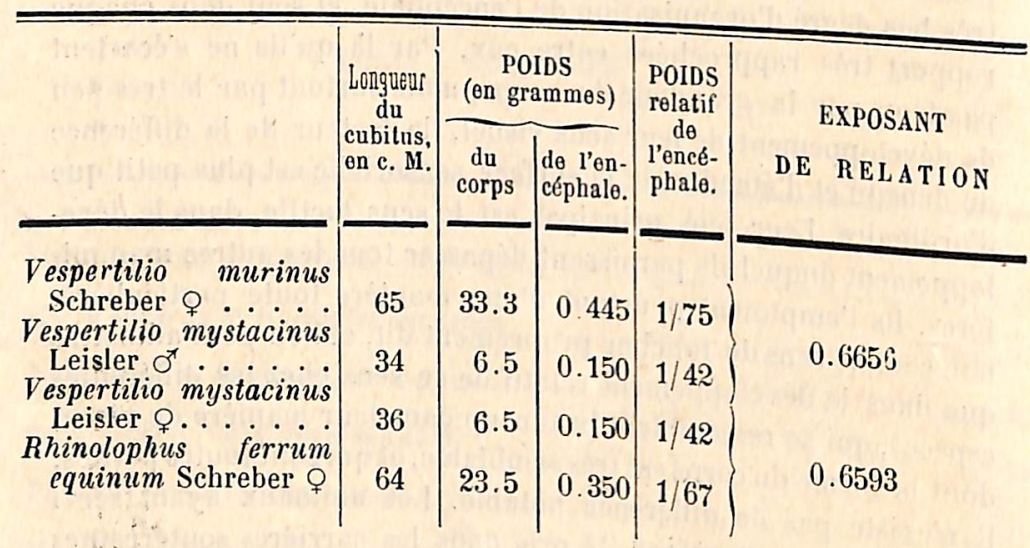

Les chiffres calculés pour l'exposant relatif sont si rapprochés de la valeur $0.6666 .$. , que $r$ obtiendrait si le poids de l'encéphale se rapportait comme les surfaces du corps, qu'il paraît déjà dé-
montré par ces deux comparaisons, qué nommés facteurs, agissant dans uns, que réellement ici les prél'autre, que c'est aussi réellement lentraire, suppriment l'un éléments sensoriels, en quelque sorte coloppement différent des dans la quantité relative du cerveau en par la différence dans les sept autres comparaisons donnaitsance blanche, qui, moindres comme résultat.

Par introduction de l'exposant relatif $r$, ainsi trouve, dans la comparaison

$$
\mathrm{e}=\mathrm{c} \cdot \mathrm{s}^{\mathrm{r}} \text { ou } \mathrm{c}=\frac{\mathrm{e}}{\mathrm{s}^{\mathrm{r}}}
$$

iff (tableIV). Proprement on devri tif(tableIV). Proprement on devrait pour certains animaux toujours
employer la valeur de $r$ trouvée pour ces pourtant encore rarement faisable ces mèmes animaux. C'es l'emploi de la valeur moyenne trouvée 0,5613 faute résultant de rable, parce que $r$ est en général peu variable n'est pas considé- ne veut qu'une évaluation approximative de la céphalisation relative.

En harmonie avec l'idée que Snell se faisait de a l'exposant somatique $\triangleright$, comme étant déterminé par l'échange de matière, il pensait que cet exposant chez tous les animaux à sang chaud pourrait être accepté comme égal. Ainsi qu'il a déjà paru et qu'il paraîtra encore dans la suite, et aussi comme il faut déduire de la signification de cet exposant, ce n'est certainement pas le cas.

On commettrait certainement des fautes plus importantes, lorsqu'on désirerait considérer le facteur de céphalisation ainsi trouvé comme une mesure du degré de l'organisation réelle de l'encéphale (donc avec Snell comme a facteur psychique $₫$ ). Les encéphales d'animaux qui appartiennent a des groupes naturels différents ne sont pas de méme valeur. Ils sont formés de parties plus et moins compliquées, d'organisation supérieure et inférieure, et augmentent chez ceux qui ont un développement supérieur non seulement en quantité, mais aussi en qualité; les parties plus compliquées deviennent de plus en plus prépondérantes et en mème temps plus fines dans leur structure intime, des animaux les plus inférieurs jusqu'aux plus élevés.

Chez les animaux supérieurs le facteur de céphalisation, désirerait-on l'interpréter comme facteur psychique ou d'organisation, serait par là généralement trop bas, et chez les animaux inférieurs trop élevé. On pourrait mieux juger ce dernier facteur, ainsi que Snell l'a proposé, en prenan ¿ pour base au lieu du poids total de l'encéphale, le poids des hémisphères cérébraux. Ceux-ci ont chez l'homme environ $4 / 50$, chez les petits animaux de l'ordre des rongeurs, les insectivores et les carnivores, environ la moitié, selon Snell, du poids total de l'encéphale. Cependant il n'existe presque pas de telles données sur le poids des hémisphères cérébraux, et aussi avec l'introduction de ce poids, au lieu du poids totai de l'encéphale, l'organisation relative réelle de l'encéphale chez des animaux différents ne pourrait être calculée, les hémisphères cérébraux eux-mèmes d'animaux d'un degré très différent d'organisation n'étant pas encore équivalents.

Il ne faut donc pas exagérer l'importance de la table suivante, généralement calculée avec 0,5613 comme valeur de $r$. Elle n'a qu'une signification très limitée pourjuger du de gré de l'organisation de l'encéphale chez des mammifères différents et ainsi du développement de leurs "fonctions psychiques ». Pour cela il

$$
\text { т. VIII ( } 4^{\circ} \text { SÉrIE). }
$$


faudrait encore considérablement hausser les chiffres très èlevés, abaisser les chiffres très inférieurs; parce qu'une organisation supérieure se manifeste aussi bien dans la qualité (macroscopiquement et microscopiquement) que dans la quantité.

Toutefois, à l'exception de quelques-uns de ces chiffres, ceux-ci sont mieux d'accord que ceux que l'on a donné déjà avec le rang que les animaux en question occupent dans le système naturel, comme aussi avec nos connaissances de leurs a facultés psychiques $\gg$.

\section{Appendice a la Table IV.}

1) D'après H. Vierordt, Daten und Tabellen für Mediciner. 2 Aufl. Jena, 1893, p. 13, 21, 22, 23. Voir aussi Th. L. W. von Bischoff, Das Hirngewicht des Menschen. Bonn, 1880, p. 143 et 152.- - Bischoff rejette lui-mèmeí(p. 27-28) la moyenne des poids du corps trouvés par lui sur des cadavres d'hommes ayant été plus ou moins longtemps lui sur des, dont les poids relatifs de l'encéphale $1 / 36$ et $1 / 35$ sont calculés (p. 31). La valeur trouvée pour $c$ chez l'homme est encore un peu trop basse, en comparaison de celle de la femme, ancore un peu spécifique plus considérable du corps de l'homme, a cause du poids relativement plus grand de son squelette thome, par suite du poids son corps en graisse.

2) Deniker et Boulart, Nouvelles Archives du Muséum, $3^{\ominus}$ série t. VII 1895, p. 56.

3) Propres déterminations des poids d'un grand individu, très âgé d'après ses dents et son squelette, tué dans l'ètat naturel.

4) J. H. F. Kohlbrugge B̈̈dragen tot de natuurlÿke

menschen en dieren. IV, p. 20 in Natuuk. Tij 55. Batavia, 1896.

D'un autre individu adulte, plus maigre, de la merese me furent communiquées les données par M. Kâme espèce, dont seraient $11156,72,1 / 155$ et 0.3850 .

5) G. Crisp in Proceed. Zool. Society of London. Part. 23. 1855, 186. 302-303.

7) Colin, Ibid., p. 303 .

8) M. Weber, Verslag van de Vergadering der Kon : Akademie v. 1896

9) Le poids de l'encéphale d'après l'éval

p. 4 (i06). Le poids du corps d'après pesagen de Weber (Vorstudien, communiqué par Weber (Ibid., p. 17 [119]). Bala William Turner, est plus lourd encore, dépassant même 100,000 aenoptera sibbaldi -
TABLE IV

\begin{tabular}{|c|c|c|c|c|c|}
\hline & \multicolumn{2}{|c|}{$\begin{array}{c}\text { POIDS } \\
\text { (en } \text { grammes) }\end{array}$} & \multirow{2}{*}{$\begin{array}{l}\text { POIDS } \\
\text { relatif } \\
\text { de } \\
\text { l'encé- } \\
\text { phale. }\end{array}$} & \multirow{2}{*}{$\begin{array}{c}\text { Facteur } \\
\text { de } \\
\text { cépha- } \\
\text { lisation }\end{array}$} & \\
\hline & du corps & $\begin{array}{l}\text { de l'en- } \\
\text { céphale. }\end{array}$ & & & \\
\hline Homo & 662001 & 1431 & $1 / 46$ & $28186 \mid \longrightarrow$ & Tovennes de 92 indiv \\
\hline Homo sapiens L. O 1 ) & $\begin{array}{l}66200 \\
54800\end{array}$ & $\begin{array}{l}1431 \\
1224\end{array}$ & $\begin{array}{l}1 / 40 \\
1 / 45\end{array}$ & $\left|\begin{array}{l}2.0100 \\
2.6778\end{array}\right|$ & Moyennes de 33 indiv. \\
\hline $\begin{array}{l}\text { Simia satyrus L. OT } \\
\text { Hylobates }\end{array}$ & $735 / 00$ & 400 & $1 / 183$ & 0.7423 & \\
\hline Desm. o & $9 \check{000}$ & 130 & $1 / 73$ & 0.7607 & \\
\hline ……… & 6250 & 94.5 & $1 / 66$ & 0.6994 & \\
\hline $\begin{array}{l}\text { Semnopithecus maurus } \\
\text { Desm. } \sigma^{\top} \cdot{ }^{1}\end{array}$ & 8800 & 70 & $1 / 126$ & 0.4276 & \\
\hline Macncus cynomolgus $\mathrm{L}$. & 0000 & 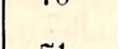 & & & \\
\hline $\begin{array}{l}\sigma^{x} \\
\text { Midas rosalia L. }{ }^{\circ}\end{array}$ & $\begin{array}{r}12000 \\
335\end{array}$ & $\begin{array}{l}71 \\
12.8\end{array}$ & $\begin{array}{l}1 / 169 \\
1 / 26\end{array}$ & $\left|\begin{array}{l}0.3636 \\
0.4897\end{array}\right|$ & \\
\hline 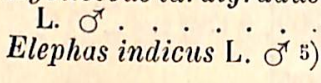 & $\begin{array}{r}500 \\
3048060\end{array}$ & ${ }^{8.18}$ & $\left|\begin{array}{l}1 / 61 \\
1 / 560\end{array}\right|$ & $\left|\begin{array}{ll}0 & 2499 \\
1.2484\end{array}\right|$ & $c=0.4000$, si $r=0.6375$ \\
\hline Equus caballus L. . 6) & 375060 & 615 & $1 / 698$ & 0.4573 & Moyennes de 30 ind. $\sigma^{x}$ \\
\hline $\begin{array}{l}\left.\text { Equus asinus L. } \bigcirc^{\top} \cdot{ }^{7}\right) \\
\text { Tapirus americanus L. }\end{array}$ & 175000 & 385 & $1 / 457$ & 0.4390 & \\
\hline Capra hircus L. . . & $\begin{array}{r}160000 \\
37500\end{array}$ & $\begin{array}{l}169 \\
124\end{array}$ & $\begin{array}{l}1 / 947 \\
1 / 302\end{array}$ & $\left|\begin{array}{l}0.2026 \\
0.3358\end{array}\right|$ & \\
\hline $\begin{array}{l}\text { Oryx beisa Rüpp. } \\
\text { Gephalophus muxwelli }\end{array}$ & 107000 & 280 & $1 / 382$ & 0.4209 & $0.331 t$ \\
\hline $\begin{array}{l}\text { H. Sm. } \\
\text { Camelopardalis giraffa }\end{array}$ & 3357 & 38 & $1 / 88$ & 0.3987 & 0.35514 si $r=0.5769$ \\
\hline $\begin{array}{l}\text { Schreb. } \sigma^{T} \text { Tragulus javanicuis Pal. } \\
\text { The }\end{array}$ & 529000 & 680 & $1 / 778$ & 0.4169 & 0.3394 \\
\hline Hippopotamus amphi- & 2037 & 15.85 & $1 / 129$ & 0.2202 & \\
\hline $\begin{array}{c}\text { bius L. } \\
\text { Balaenoptera } \\
\text { sibbaldi }\end{array}$ & 1755000 & 582 & 1/3015 & 0.1819 & \\
\hline $\begin{array}{l}\left.\text { Mustela putorius } \mathrm{L} .{ }^{10}{ }^{10}\right) \\
\text { Lycaon pictus } \mathrm{Temm}\end{array}$ & 74000000 & 7000 & $1 / 76$ & 0.2166 & $\begin{array}{l}c=0.2263 \text { si } s=100000 \\
\text { K.G. (poids rel. de l'en- } \\
\text { céphale }=\frac{1}{14280} \text { ) }\end{array}$ \\
\hline $\begin{array}{c}\sigma^{\top} \\
\text { Canis familiarislappon. }\end{array}$ & 25400 & 131 & $1 / 193$ & 0.4413 & très vioux. \\
\hline $\begin{array}{l}\text { OTa } \\
\text { Canis familiaris Bernh. }\end{array}$ & 12040 & 70 & $1 / 72$ & 0.3586 & vieux. \\
\hline $0^{1}$. & 46000 & 123 & $1 / 373$ & 0.2970 & \\
\hline $\begin{array}{l}\text { Ganis famitiaris Bernh. } \\
\left.\sigma^{\top} . \cdots \cdot \ldots \cdot 11\right)\end{array}$ & 53000 & 123 & $1 / 430$ & 0.2743 & \\
\hline
\end{tabular}




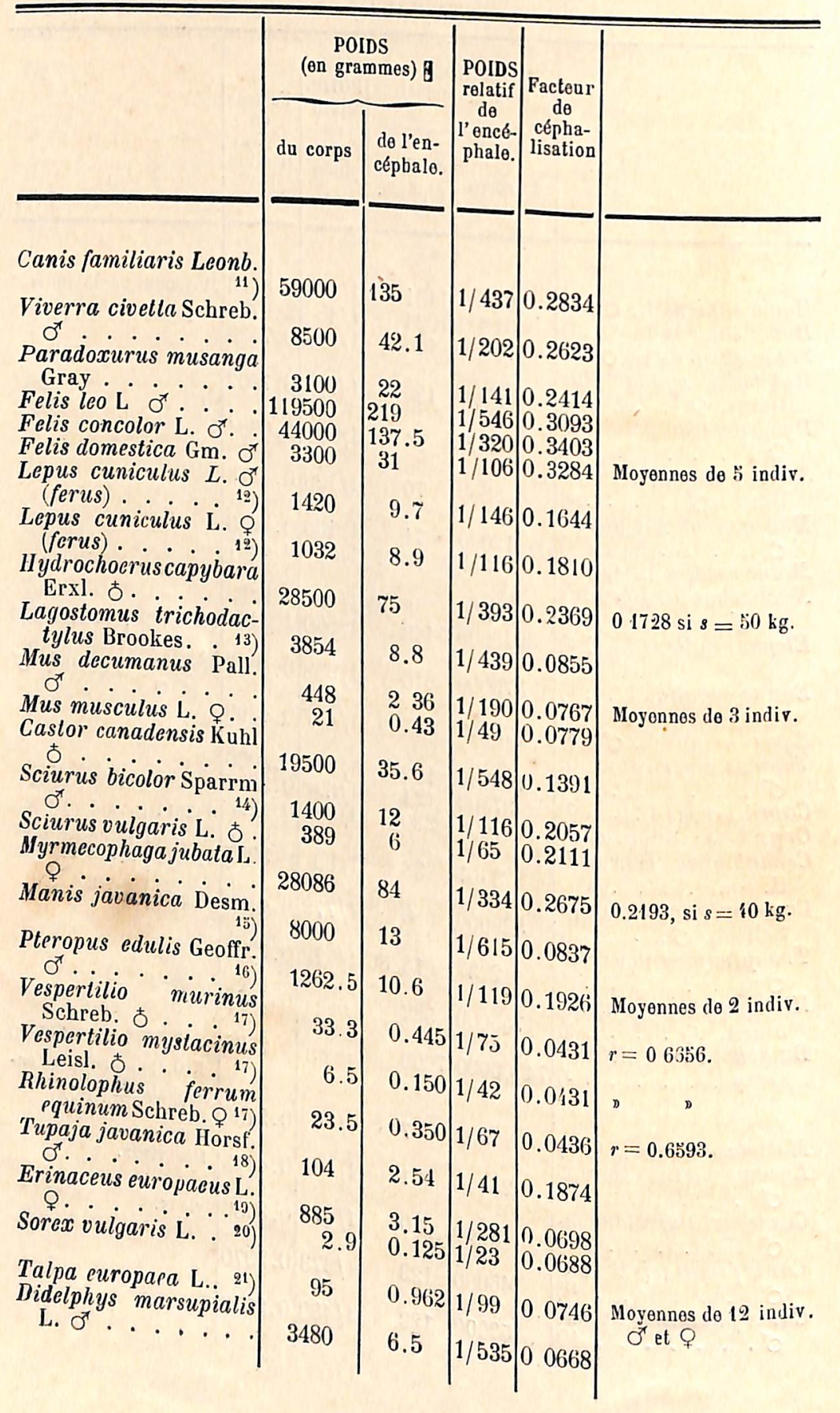

drait aussi mettre hors de compte les $30,000 \mathrm{~kg}$. de lard, dont est couvert le corps de ces mammifères.

10) Propres pesages d'un individu adulte. Longueur du tronc avec la tête $35 \mathrm{c} . \mathrm{m}$.

11) Rüdinger in Verhandlungen Anatom. Gesellschaft. Jena 1894, p. 173, Cf. Weber I. c. p. 9 (111).

12) Propres pesages d'animaux, ètant adultes d'après le squelette.

13) R. Owen in Proceed. Zool. Soc. of London. Part. 7, 1839, p. 175.

14) Kohlbrugge, loc. cit., p. 37.

15) Kohlbrugge, loc. cit., p. 35

16) Kohlbrugge, loc. cit., p. 38 et Weber, Vorstudien, p. 7 (109).

17) Propres pesages.

18) Kohlbrugge, l. c. p. 38 et Weber, Vorstudien, p. 6 (108).

19) Propres pesages.

20) O. Snell, Archiv für Psychiatrie. I3d. 23, 1891, p. 443 et 445. Le poids du corps est calcule d'après le poids absolu et relatif de l'encéphale communiqués par lui. Ces chiffres sont si bas que probablement ils ont rapport à Sorex minutus L. et non à Sorex vulgaris L.

21) L. Manouvrier, sur l'interprétation de la quantité dans l'encéphale, loc. cit., p. 297.

Les autres données sont empruntées aux Vorstudien de Weber, qu mentionne aussi celles sous les numéros 2), 5), 11), 13), 14), 15), 16) et 18).

Il parait que Hydrochœrus et Myrmecophaga peuvent devenir plus lourds sans que le poids de l'encéphale augmente. C'est pour cela que pour eux $c$ est calculé deux fois différemment.

Dépassant mème environ quatre fois en vraie quantité relative de l'encéphale les singes anthropoïdes, l'homme occupe une place très élevée au-dessus de tous les autres mammifères. Les singes anthropoïdes ont seulement environ une quantité double d'encéphale que les carnivores el les ruminants, au-dessus desquels s'élèvent à peine les singes inférieurs. Les carnivores et les ongulés sont à peu près égaux. Les poids relativement bas du Kanchil, de l'hippopotame et des cétacés résultent peul-ètre de ce qu'ils sont des formes très anciennes. L’hippopotame et la baleine géante, qui d'après leur poids relatif de l'encéphale occupent une place fort inférieure, sont néanmoins considérablement élevés, si on les considère du point de vue, auquel je me suis placé dans ce travail, et se rangent près des rongeurs. Ceux-ci sont en parlie beaucoup en dessous des ongulés et des carnivores; il vaudrait certes la peine de chercher quelle est la cause des différences re- 
marquables qu'on observe dans ce groupe. Les insectivores, le pangolin et les marsupiaux occupent, au point de vue du poids de leurs encéphales, une situation encore inférieure à celle desrongeurs. Le tamanoir est cependant considérablement supérieur au pangolin, une nouvelle raison pour séparer les édentés américains de ceux de l'Ancien Monde. La roussette s'écarte portion considérable des mis en pro insectivores), qui de trouvent trouvent au dernier degré pour ce qui concerne la quantité
relative de l'encéphale.

Plus bas encore se trouvent toutefois les mammifères de l'époque du Phenate d'un moulage de la cavité cranienne rain éocène, le poids de l'ancêtre du cheval du plus ancien termativement à tapir, et ainsi on trouve pour animal était environ de la taille du surtout petite quand on pour $c$ une valeur de 0.0420 , qui paraît l'encéphale en question chez les chauve-souris ou touteau avait moins de quote-part que lement vivante. C'est le Dinoceras espèce de mammifères actueléocène qui, de tous les mamoceras mirabile de l'ancien terrain la plus petite quantité d'encéphères disparus et récents, présente corps. D'après le moulage de la en rapport avec le poids du à 92 grammes le poids de l'encéphité crânienne on peut évaluer taille de l'hippopolame, et animal qui avait la tié de la valeur du Phenacodus ne trouve alors pour $c$ que la moiUne situation

occupée par l'éléphant. La quantité des autres mammifères est celui-ci serait environ trois foencéphale trouvée pour ruminants. Cela n'est d'accord aussi grande que chez les occupe dans le système zoologique, ni avec le rang que l'éléphant facultés psychiques - quoiqu'o, ni avec ce que l'on sait de ses La cause de ce résultat évidemmen ait une très bonne opinion. $c$ doit. je pense être l'évaluation de l'éléphant devrait être plus grande valeur donnée, à $r$, qui pour volutions cérébrales plus grande. Car l'influence des circonun cerveau de telles dimensions, valoir qu'il ne faudrait pour Ainsi l'influence directe de la tén d'après ce que l'on voit ailleurs. brale peut se faire valoir d'une lénuilé constante de l'écorce cérédérable. Quoique l'encéphale de l'éce exaordinairement consi- que celui du bœuf ${ }^{1}$ il ne le dépasse pas très considérablement par la richesse des circonvolutions, et est sous ce rapport certes inférieur à celui des cétacés, même des plus petits, comme le dauphin. Le cerveau de l'éléphant est donc moins riche en substance grise, en comparaison avec d'autres mammifères, qu'on ne le déduirait du poids de l'encéphale, et relativement plus riche au contraire en substance blanche, par le fait que les fibres médullaires, dont celle-ci se compose, sont devenues relativement plus longues. Il faut en rechercher la cause dans l'accroissement secondaire du cerveau par suite de l'agrandissement excessif que le crâne a subi. Celui-ci a pris, grâce aux cavités aériennes très larges dans le diploë de presque tous ses os, des dimensions vraiment énormes par rapport au reste du corps. Sans doute, cela est une suite de l'élargissement de la surface d'implantation pour les muscles de la trompe, qui chez l'éléphant n'est pas seulement l'organe de l'odorat et du toucher, mais aussi un instrument très adroit et puissant de préhension; dans cette fonction la trompe est pour l'éléphant la lèvre, le doigt, la main et le bras réunis. Or il parait que la cavité crânienne aussi, cédant, aux exigences de cet organe important, a suivi l'accroissement extérieur du crâne, et que par là l'encéphale a été très agrandi. Cet agrandissement passif de l'encéphale ne pouvait tou tefois ètre atteint que par l'allongement et l'épaississement des gaînes, fonctionnellement indifférents des fibres médullaires de la substance blanche, ce quiḷn'imposa pas à l'organisme de grandes dépenses en matériel, parce que l'activité d'échange de matière de ces fibres est très faible. Le volume de la substance grise, le numbre des cellules nerveuses et la ramification de leurs prolongements n'augmentaient pas par là. Pour évaluer le facteur de céphalisatiun de l'éléphant en comparaison avec les autres mammifères, il faut donc donner à l'exposant de relation $r$ une plus grande valeur. Il parait qu'on trouve le facteur de céphalisation environ égal à celui des ruminants, donc égal à 0.4000 , si l'on pose $r=0.6375$, qui est beaucoup moins différent de $0.6666 .$. , que la valeur moyenne trouvée 0.5613 , avec laquelle dans la table $c$ est calculée aussi chez l'éléphant.

C'est au plissement relativement très abondant de l'écorce cérébrale qu'il me semble falloir attribuer le chiffre inférieur trouvé

${ }^{1}$ Voir les poids de l'encéphale des bœufs chez Robin, l. c. Le poids du corps du bœuf est aussi environ seulement la dixième partie də celui de l'éléphant. 
pour Nycticebus. Tandis que, chez les plus petits singes, la surface du cerveau est presque lisse, on la trouve chez ce lémurien et ses proches parents relativement très plissée, quoique la grandeur du corps soit à peu près la mème.

Le cheval et l'ane ont, en comparaison avec d'autres ongulés, un assez haut chiffre pour la quantité relative de leur encéphale. Cela peut cependant provenir de ce qu'on no vient à examiner ces animaux que quand ils sont très maigres et dépéris. A la mème cause on peut bien altribuer aussi le poids relatif plus élevé de l'encéphale des chiens qualifiés par Weber comme « vieux» et " très vieux ". Au contraire le rat et la souris, de ce qu'ils pourvoient facilement à leur existence, auront obtenu de grands poids du corps et ainsi de faibles poids relatifs de l'encéphale.

Je pense qu'il faut mettre en rapport avec les phénomènes dont il est parlé ici, le fait remarquable que le cerveau des échidnés, quoique ces animaux ne soient pas plus grands que l'ornithorinque, est cependant beaucoup plus riche en circonvolutions que celui de ses proches parents Chez les échidnés l'encéphale semble avoir dù s'adapter à un cràne plus petit que chez l'ornithorinque, par suite de la surface d'insertion beaucoup moindre nécessaire pour l'attache des muscles masticateurs, noindre né temporaux. Une réduction de volume de l'encéphale, sans que sa fonction reste en arrière, n'est possible que par raccourcissement des fibres, peut-ètre combiné ovec épaississement de leurs gaines médullaires, de la substance blanche, qui par là diminue en volume; l'écurce cérébrale n’étanl pas diminuée doil alors se plisser. Il semble bien certain que l'encéphale ne s'ail alors se toujours activemenl, mais que leelcéphale ne s'agrandit pas vement le crane, ce qu'il que celui-ci doil suivre parfois passilors d'une apprécialiou pas non plus perdre de vue M. Manouvraer dit qu'ia quantité de l'encé,

valuation de la supériorité traité le premier la question d'é$1881^{2}$, puis dans dans liencéphale (1883), elc. 3 in sur l'interpritation de la quanticí sur l'intéressant travail de M. Dubuis.

grèle en prrticulier. Bull. Soc cérébraux des lémuriens en général et du loris

2 L. Manouvier : C. de l'Acad. des scien Paris, 1895, t. VI, fasc. 4.

3 Sur un procidé d'analyse du pos sciences.

Att. cerveau du Dict. de Plysiol, de Cérébral. (Soc. de Biologie, 1891). -

G67 SÉANCE. - \ Juillet 1897.

Transformisme et son interprétation en craniologie

Par M. G. Papillault

Avant d'aborder le sujet spécial de cette conférence, je tiendrais a faire comme une introduction qui nous sera très utile pour bien comprendre la craniologie, en disant tout d'abord quelques mots sur le transformisme lui-mème, et en expliquant pourquoi la Société d'Anthropologie rend hommage à cette doctrine et semble l'identifier avec la science mème.

La zoologie, dont l'anthropologie n'est qu'une partie, demande, comme toute science, pour ètre bien comprise, à ètre envisagée sous deux points de vue : la méthode et la doctrine. Seule, en effet, la méthode conduirait à un formalisme creux, et la doctrine c'est-ì-dire l'ensemble des résultats, étudiée sans qu'on ait compris la méthode générale, conduirait à un empirisme grosi ner complexité des phénomènes vitaux. Or le transformisme, comme je le comprends, n'est pelles lois découvertes par la science pas seulement une des plus belles lós d'étude, absolument némoderne, c'est avant to fertile en découvertes futures.

Quand la science n'était encore qu'à ses premiers débuts, alors qu'on avait constaté que certains phénomènes, comme ceux de l'acoustique, obéissent à des rapports numériques, un bel enthousiasme s'emparait des esprits. Pythagore répétait que tout était nombre et Platon n'hésitait pas à expliquer l'univers avec sa théorie des nombres. Au fond, c'était la raison humaine qui prenait conscience d'elle-même, et qui, avec la belle confiance des premiers ages, affirmant l'idendité des lois logiques et des lois naturelles, manifestait, dès ses premiers pas, son besoin d'unifier quantitativement la variété desis phénomènes, et de fondre en une 